AperTO - Archivio Istituzionale Open Access dell'Università di Torino

\title{
Long term experience and performance of COMPASS RICH-1
}

\section{This is the author's manuscript}

Original Citation:

Availability:

This version is available http://hdl.handle.net/2318/1619342

since 2016-11-30T19:12:17Z

Published version:

DOI:10.1088/1748-0221/9/09/C09011

Terms of use:

Open Access

Anyone can freely access the full text of works made available as "Open Access". Works made available under a Creative Commons license can be used according to the terms and conditions of said license. Use of all other works requires consent of the right holder (author or publisher) if not exempted from copyright protection by the applicable law. 
Long term experience and performance of COMPASS RICH-1

This content has been downloaded from IOPscience. Please scroll down to see the full text.

\section{JINST 9 C09011}

(http://iopscience.iop.org/1748-0221/9/09/C09011)

View the table of contents for this issue, or go to the journal homepage for more

Download details:

IP Address: 192.84.137.37

This content was downloaded on 30/11/2016 at 18:11

Please note that terms and conditions apply.

You may also be interested in:

Status and progress of novel photon detectors based on THGEM and hybrid MPGD architectures M Alexeev, R Birsa, F Bradamante et al.

MPGD-based counters of single photons developed for COMPASS RICH-1

M Alexeev, R Birsa, M Bodlak et al.

Development of THGEM-based photon detectors for Cherenkov Imaging Counters

M Alexeev, M Alfonsi, R Birsa et al.

Detection of single photonswith ThickGEM-based counters

M Alexeev, F Barbosa, R Birsa et al.

Ion backflow in thick GEM-based detectors of single photons

M Alexeev, R Birsa, F Bradamante et al.

The electromagnetic calorimeter for the T2K near detector ND280

D Allan, C Andreopoulos, C Angelsen et al.

Development of high resolution tracking detectors with Gas Electron Multipliers

L I Shekhtman, V M Aulchenko, V S Bobrovnikov et al.

Performance of the ATLAS Liquid Argon Calorimeter after three years of LHC operation and plans for a future upgrade

P Strizenec

Analysis and correction of the magnetic field effects in the Hybrid Photo-Detectors of the RICH2

Ring Imaging Cherenkov detector of LHCb.

R Cardinale, C D'Ambrosio, R Forty et al. 


\section{Long term experience and performance of COMPASS $\mathrm{RICH}-1$}

F. Tessarotto, ${ }^{a}{ }^{1}$ P. Abbon,,${ }^{b}$ M. Alexeev, ${ }^{a}$ R. Birsa, ${ }^{a}$ P. Bordalo, ${ }^{c}$ F. Bradamante,${ }^{d}$

A. Bressan, ${ }^{d}$ M. Büchele, ${ }^{e}$ M. Chiosso,${ }^{f}$ P. Ciliberti, ${ }^{d}$ T. Dafni, ${ }^{b}$ S. Dalla Torre, ${ }^{a}$

S. Dasgupta, ${ }^{a}$ E. Delagnes, ${ }^{b}$ O. Denisov, ${ }^{g}$ V. Duic, ${ }^{d}$ A. Ferrero, ${ }^{b}$ M. Finger, ${ }^{h}$

M. Finger Jr., ${ }^{h}$ H. Fischer, ${ }^{e}$ C. Franco, ${ }^{c}$ S. Gerassimov, ${ }^{i}$ B. Gobbo, ${ }^{a}$ M. Gregori, ${ }^{a}$

F. Herrmann, ${ }^{e}$ B. Ketzer, ${ }^{j}$ K. Königsmann, ${ }^{e}$ I. Konorov,${ }^{i}$ F. Kunne,${ }^{b}$ S. Levorato, ${ }^{a}$

A. Maggiora, ${ }^{g}$ N. Makke, ${ }^{d}$ A. Martin, ${ }^{d}$ G. Menon, ${ }^{a}$ D. Neyret, ${ }^{b}$ K. Novakova, ${ }^{a, l}$

D. Panzieri, ${ }^{k}$ S. Paul ${ }^{i}$ F.A. Pereira, ${ }^{a}$ J. Polak, ${ }^{a}$ E. Rocco,${ }^{a}$ C.A. Santos,${ }^{a}$ G. Sbrizzai, ${ }^{d}$

P. Schiavon, ${ }^{d}$ S. Schopferer, ${ }^{e}$ M. Slunecka, ${ }^{f}$ F. Sozzi, ${ }^{a}$ L. Steiger $,{ }^{a}, l$ M. Sulc ${ }^{l}$ and S. Takekawa ${ }^{g}$

\footnotetext{
${ }^{a}$ INFN, Sezione di Trieste, Trieste, Italy

${ }^{b}$ CEA Saclay, DSM/DAPNIA, Gif-sur-Yvette, France

${ }^{c}$ LIP and Instituto Superior Técnico, Universidade de Lisboa, Lisbon, Portugal

${ }^{d}$ INFN, Sezione di Trieste and University of Trieste, Trieste, Italy

${ }^{e}$ Universität Freiburg, Physikalisches Institut, Freiburg, Germany

${ }^{f}$ INFN, Sezione di Torino and University of Torino, Torino, Italy

${ }^{g}$ INFN, Sezione di Torino, Torino, Italy

${ }^{h}$ Charles University, Prague, Czech Republic and JINR, Dubna, Russia

${ }^{i}$ Technische Universität München, Physik Department, Garching, Germany

${ }^{j}$ Rheinische Friedrich-Wilhelms-Universität Bonn, Bonn, Germany

${ }^{k}$ INFN, Sezione di Torino and University of East Piemonte, Alessandria, Italy

${ }^{l}$ Technical University of Liberec, Liberec, Czech Republic

E-mail: fulvio.tessarotto@ts.infn.it
}

\footnotetext{
${ }^{1}$ Corresponding author.
} 
ABSTRACT: COMPASS RICH-1 is a large size gaseous Imaging Cherenkov Detector providing hadron identification in the range from 3 to $55 \mathrm{GeV} / c$, in the wide acceptance spectrometer of the COMPASS Experiment at CERN SPS.

It uses a $3 \mathrm{~m}$ long $\mathrm{C}_{4} \mathrm{~F}_{10}$ radiator, a $21 \mathrm{~m}^{2}$ large VUV mirror surface and two kinds of photon detectors: MAPMTs and MWPCs with CsI photocathodes, covering a total of $5.5 \mathrm{~m}^{2}$. It is in operation since 2002 and its performance increased thanks to progressive optimization and to a major upgrade of its photon detection system, implemented in 2006; a new upgrade is foreseen for 2016, with the use of MPGD-based photon detectors.

The main characteristics of COMPASS RICH-1 components are described and the most critical aspects related to the $\mathrm{C}_{4} \mathrm{~F}_{10}$ radiator gas system, to the mirrors and their alignment, as well as the performance of the photon detectors are presented and discussed. The response of the MWPCs and the observed evolution of the effective quantum efficiency of the CsI photocathodes is analyzed. The properties and performance of the MAPMTs with individual fused lens telescopes are presented together with the readout characteristics. The PID performance of COMPASS RICH-1 is discussed and the future upgrade program is mentioned.

KEYwORDS: Particle identification methods; Cherenkov detectors; Photon detectors for UV, visible and IR photons (gas); Photon detectors for UV, visible and IR photons (vacuum) 


\section{Contents}

1 Introduction 1

2 The RICH-1 vessel and the gas radiator 3

3 The mirrors and the CLAM system 6

4 The MWPC-based photon detectors 10

5 The MAPMT-based photon detectors, the readout and DAQ 14

$\begin{array}{llr}6 & \text { PID performance } & 17\end{array}$

$\begin{array}{lll}7 & \text { Perspectives and conclusions } & 19\end{array}$

\section{Introduction}

The COMPASS Experiment [1] at CERN has performed between 2002 and 2011 an extensive program of measurements to investigate hadron structure and hadron spectroscopy.

It used the high energy SPS muon beam and a large polarized target to perform measurements of proton and deuteron spin structure (transversity, gluon polarization, flavor helicity decomposition, transverse momentum dependent PDFs, etc.). With SPS hadron beams it measured pion polarizability, hadron diffractive and central production, light meson spectroscopy, baryon spectroscopy, etc.

A new series of ambitious measurements [2] for the period between 2012 and 2018 has been approved: Drell-Yann studies as well as pion and kaon polarizability measurements are performed with hadron beams, while Deeply Virtual Compton Scattering and Exclusive Meson Production reactions as well as Semi-Inclusive Deep Inelastic Scattering reactions will be studied with the high intensity muon beam.

The COMPASS apparatus (see figure 1) consists of a two-stage, large angle and large momentum acceptance spectrometer [3], providing high rate capability, high time and space resolution, good calorimetry and excellent Particle Identification (PID).

To provide hadron identification in a very large momentum range two gaseous RICH counters were originally foreseen in the COMPASS proposal [1] (see figure 2); the construction of COMPASS RICH-2 has however been postponed while RICH-1 (see figure 3) has successfully been implemented and improved in order to meet the challenging COMPASS PID requirements.

The RICH-1 detector [4-7] uses a $3 \mathrm{~m}$ long gaseous $\mathrm{C}_{4} \mathrm{~F}_{10}$ radiator, a $21 \mathrm{~m}^{2}$ large focusing VUV reflecting surface and two kinds of photon detectors (PDs): MAPMTs and MWPCs with CsI photocathodes, placed outside of the acceptance and covering a total surface of $5.5 \mathrm{~m}^{2}$. 
It provides $\pi$-K separation from 3 to $55 \mathrm{GeV} / c$ over an angular acceptance of $\pm 250 \mathrm{mrad}$ in the horizontal plane and $\pm 180 \mathrm{mrad}$ in the vertical plane, with beam rates up to $40 \mathrm{MHz}$ and trigger rates up to $20 \mathrm{kHz}$.

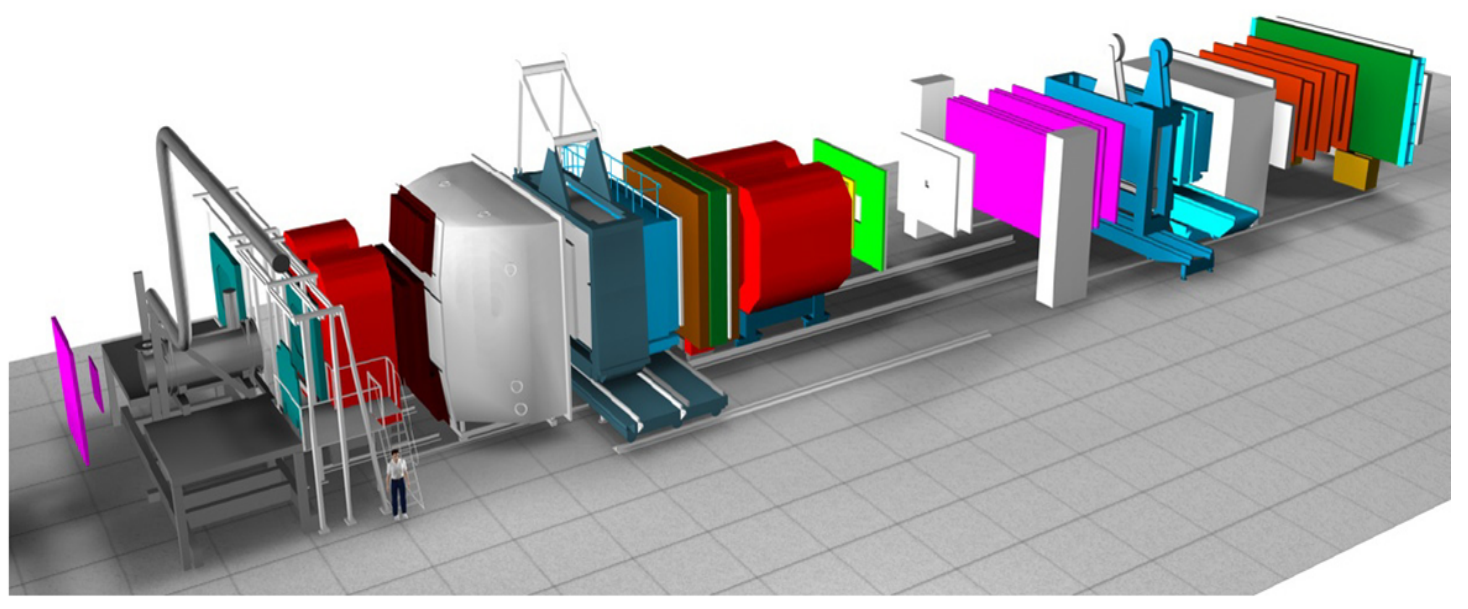

Figure 1. Artistic view of the COMPASS spectrometer; in red the two dipole magnets; in grey the RICH-1 detector.

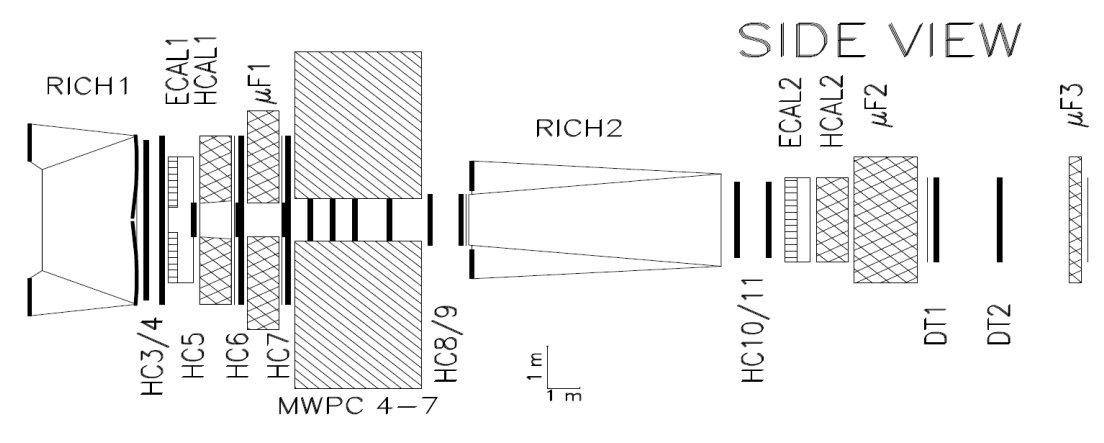

Figure 2. Part of the COMPASS set-up from the Proposal of the Experiment: two RICH counters were foreseen.

The RICH-1 detector has been designed in 1996 and it is in operation since 2002; its performance has increased in time thanks to progressive optimization and mostly to a major upgrade which was implemented in 2006.

For the new COMPASS measurements an upgraded version of the apparatus is being prepared; in the Particle Identification (PID) sector increased rate capability, greater stability and higher efficiency need to be guaranteed; a new upgrade of the gaseous photon detectors of RICH-1 is foreseen for 2016.

This article describes the main components of COMPASS RICH-1, their implementation, improvement and present performance. In section 2 the characteristics of the RICH-1 vessel, of the radiator gas and of the gas system are presented; in section 3 the properties of the mirror wall are described and the mirror alignment issue is discussed; in section 4 the original Photon Detectors 


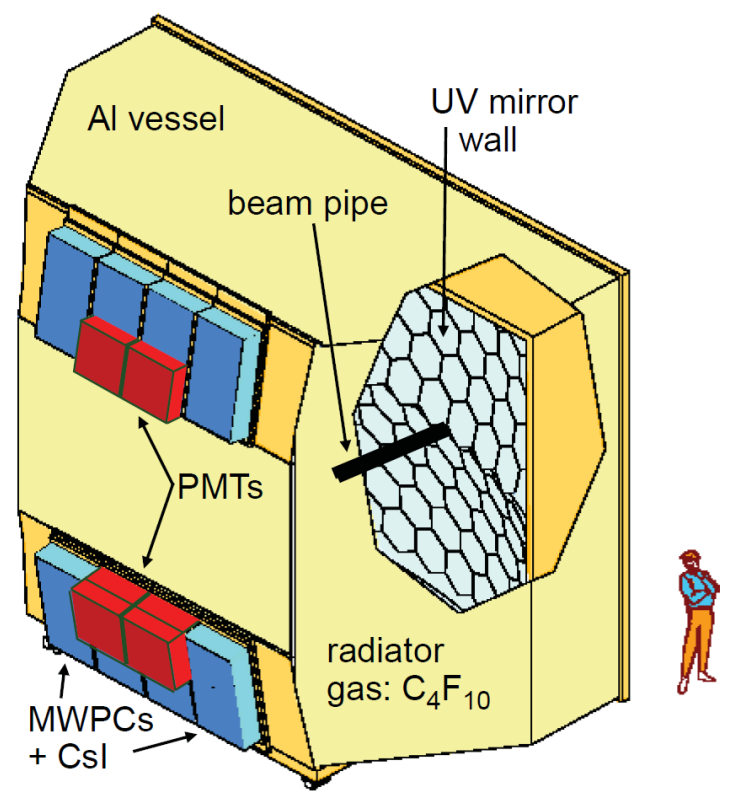

Figure 3. Artistic view of COMPASS RICH-1.

(PDs), based on the use of MWPCs and CsI, are introduced, their performance is discussed and the results from the analysis of long term CsI QE variations are presented in detail; section 5 is dedicated to the PDs covering the central region of RICH-1, based on the use of MAPMTs and to a short presentation of the RICH-1 readout and DAQ systems. The main feature of the data analysis and the PID performance of RICH-1 are summarized in section 6 . Section 7 briefly describes the new MPGD-based PDs which will be implemented in the foreseen upgrade of COMPASS RICH-1.

\section{The RICH-1 vessel and the gas radiator}

Hadron identification in the high momentum domain imposes the use of a gas radiator; the choice of MWPCs with CsI photocathodes (the only affordable technology for covering large photosensitive surfaces at that time), coupled to the specific COMPASS spectrometer characteristics (in particular the large acceptance) lead to the need of a large size radiator volume (about $80 \mathrm{~m}^{3}$ ).

The RICH vessel (figure 4) consists in an aluminum structure closed by Al plates; it has a length of $3 \mathrm{~m}$, a width of $6 \mathrm{~m}$ and a height of about $5 \mathrm{~m}$. The front and rear windows need to hold the radiator gas hydrostatic pressure with a minimum amount of material, being in the spectrometer acceptance: each one is a sandwich of two aluminum foils and a layer of rigid foam, for a total of $2 \% \mathrm{X}_{0}$. In the central region, where the beam is passing, their contribution to the material budget is reduced to $0.6 \% \mathrm{X}_{0}$ each.

Interfacing the vessel with the windows, the support frames for the photon detectors and the other elements via o-rings could be achieved with minimal gas leaks and mechanical stresses thanks to the accurate machining and polishing of all vessel contact frames and to the tight mechanical tolerances imposed to the production of all components. The leakage rate is lower than $3 \mathrm{~Pa} \cdot l / s$.

To reduce the amount of material in the beam region $\left(\sim 10 \% \mathrm{X}_{0}\right.$ from the radiator gas $)$ and to protect the RICH from the large amount of Cherenkov photons generated by the beam particles 


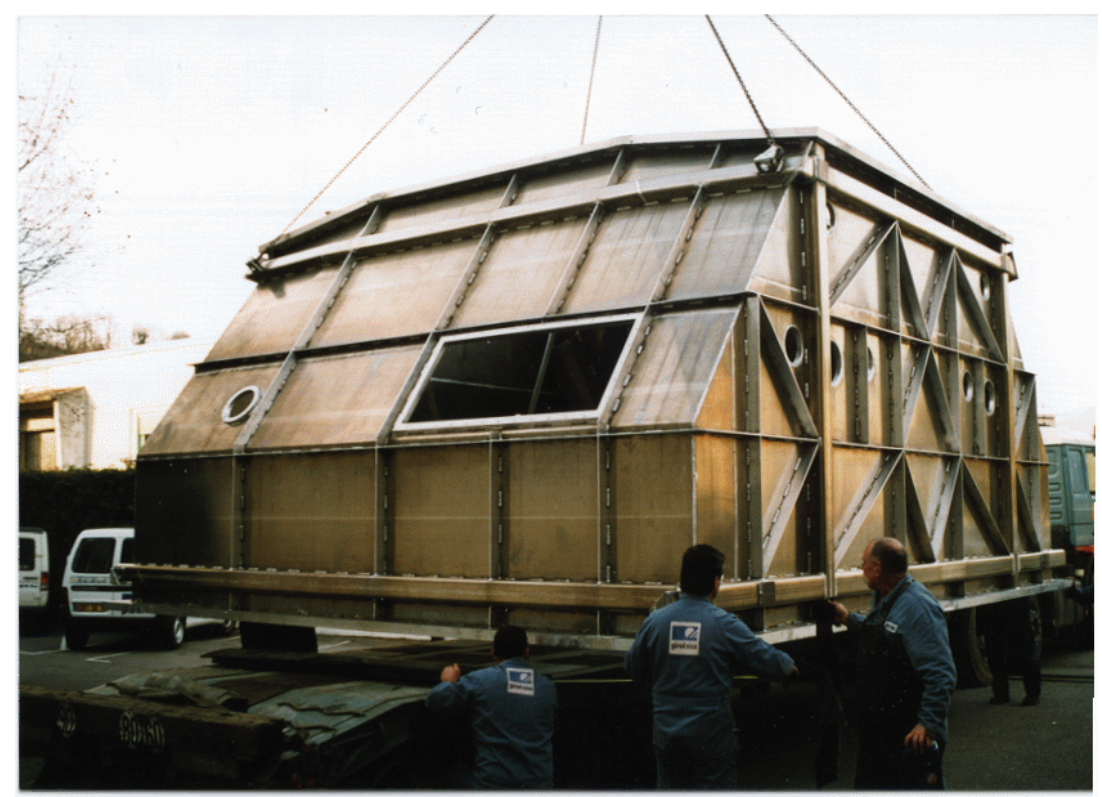

Figure 4. Picture of the RICH-1 vessel during transport.

a cylindrical pipe of $100 \mathrm{~mm}$ diameter has been installed (see figure 5) and filled with helium. The original beam pipe, made of $150 \mu \mathrm{m}$ thick stainless steel, has been replaced in 2012 by a lighter pipe, (see figure 6) made of 4 layers of metalized BoPET $(25 \mu \mathrm{m}$ BoPET $+0.2 \mu \mathrm{m} \mathrm{Al}$ ): the contribution to the total material budget by the new pipe for beam particles is $0.08 \% \mathrm{X}_{0}$ (plus $0.06 \%$ due to helium).
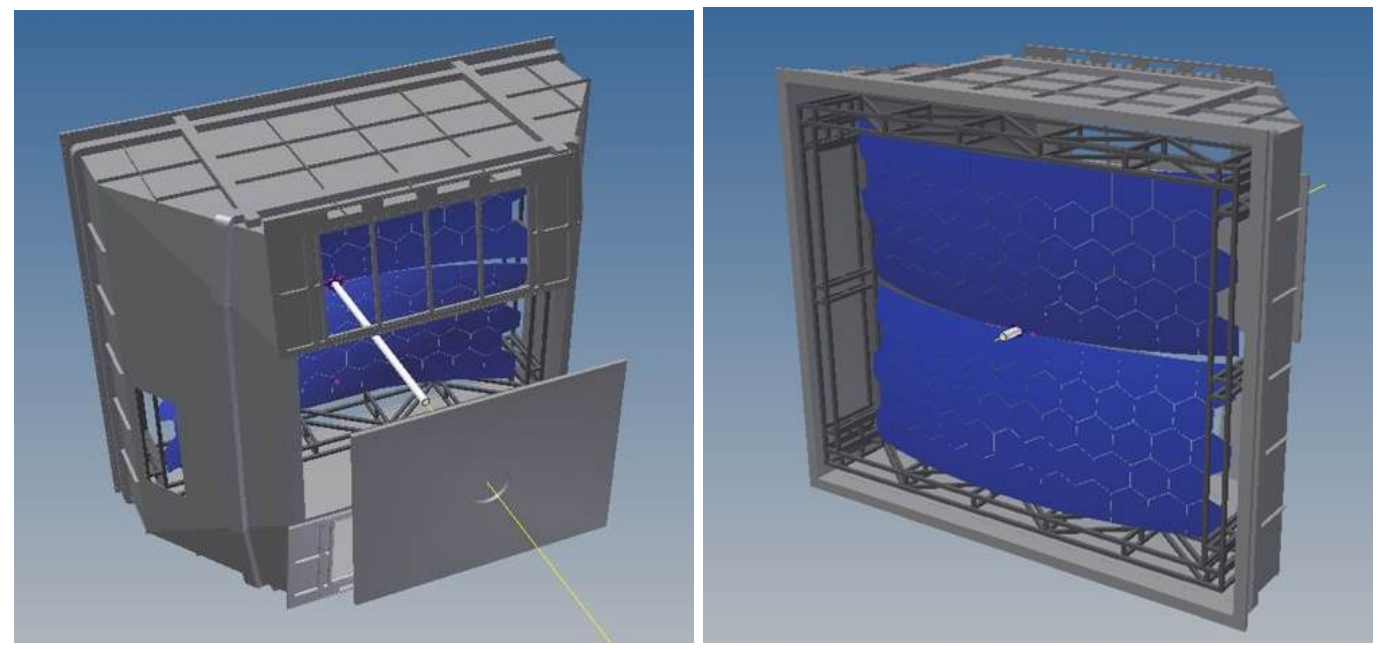

Figure 5. Two views of the opened RICH-1 vessel with the mirrors in blue and the beam pipe in white.

The choice of the radiator gas, $\mathrm{C}_{4} \mathrm{~F}_{10}$, the heaviest fluorocarbon in gaseous phase at STP, was dictated by the need to guarantee both low Cherenkov thresholds $(2.5,9,17 \mathrm{GeV} / c$ for $\pi, \mathrm{K}, \mathrm{p}$ respectively) and low chromatic dispersion. To avoid excessive stress on the vessel windows the gas is kept at atmospheric pressure (plus $100 \mathrm{~Pa}$ with a $10 \mathrm{~Pa}$ maximum allowed deviation at a 


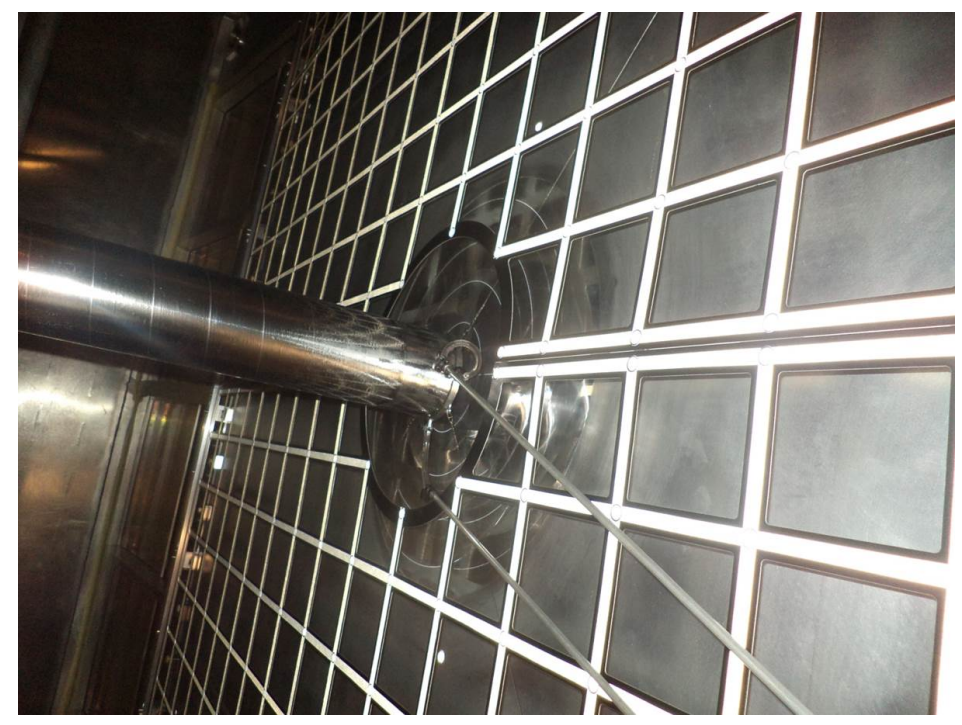

Figure 6. Picture of a part of the light beam pipe inside RICH-1.

reference point, on the top of the vessel). A dedicated gas system [8] continuously circulates the gas in a closed loop circuit at a rate of about $2 \mathrm{~m}^{3} / \mathrm{h}$.

To ensure a high transmittance for Cherenkov photons the contamination from UV absorbing impurities has to be minimized: $\mathrm{Cu}$ catalyst filters (operated at $40^{\circ} \mathrm{C}$ ) and $5 \mathrm{~A}$ molecular sieves (at $15^{\circ} \mathrm{C}$ ) are used to keep water vapour and oxygen contamination levels respectively below 1 ppm and 3 ppm.

A storage tank keeps the $\mathrm{C}_{4} \mathrm{~F}_{10}$ in liquid phase before and after the COMPASS running periods; during normal operation the compressors of the gas system suck the $\mathrm{C}_{4} \mathrm{~F}_{10}$ from the vessel at a fixed rate and force it through the filters and a liquefier to the storage tank, where, after evaporation it is sent back to the vessel at the rate needed to exactly compensate for compressor sucking, atmospheric pressure variations and leaks, which are typically at the level of $60 \mathrm{l} / \mathrm{day}$.

The vessel has to be kept at atmospheric pressure during the $\mathrm{C}_{4} \mathrm{~F}_{10}$ filling and recovery operations too, at the beginning and at the end of the running periods: efficient separation between $\mathrm{N}_{2}$ and $\mathrm{C}_{4} \mathrm{~F}_{10}$ is obtained by operating the liquefier at a pressure larger than 6 bars and at a temperature lower than $-35^{\circ} \mathrm{C}$, where the equilibrium vapour pressure of $\mathrm{C}_{4} \mathrm{~F}_{10}$ is $\leq 20 \mathrm{kPa}$.

The levels of $\mathrm{O}_{2}$ and $\mathrm{H}_{2} \mathrm{O}$ contamination and the total amount of $\mathrm{C}_{4} \mathrm{~F}_{10}$ in the system are continuously monitored while the transparency is frequently checked by measuring the light transmission in an automated system based on a $\mathrm{D}_{2}$ lamp, a monochromator and UV sensitive PMs. The stability of the radiator gas mixture (typically: $\mathrm{C}_{4} \mathrm{~F}_{10} / \mathrm{N}_{2}=97 / 3$ ) is measured by a sonar-based system in the same set-up. A system providing an accurate measurement of the radiator gas refractive index based on the use of a modified Jamin interferometer is presently under preparation [9]

Light transmission values larger than $88 \%$ in the VUV wavelength domain of the CsI-based photon detectors $(165-210 \mathrm{~nm})$, close to the limit provided by Rayleigh scattering process, are routinely obtained: a typical example of a radiator transparency measurement result can be seen in figure 7 where the transparency of $187 \mathrm{~cm}$ of the gas as function of the wavelength is checked and fitted to extract the estimated contamination levels for the UV absorbing impurities. 
A specific cleaning procedure needs to be applied before usage to the commercially available $\mathrm{C}_{4} \mathrm{~F}_{10}$, which is full of impurities [10]: a system in which the $\mathrm{C}_{4} \mathrm{~F}_{10}$ circulates in closed loop through activated carbon and $3 \mathrm{~A}$ molecular sieves and condenses in a cold section where the gas component is vented out has been built for this purpose; the typical fraction of material lost in this cleaning operation varies between 0.1 and 0.3 .

A complete temperature stabilization of COMPASS RICH-1 has not yet been implemented, but a dedicated system providing up to $20 \mathrm{~m}^{3} / \mathrm{h}$ circulation flow between the top and the bottom of the vessel has been implemented to avoid the formation of thermal or composition gradients in the radiator gas volume.

During the entire running period regular checks and immediate emergency interventions are granted by COMPASS RICH-1 experts on call.

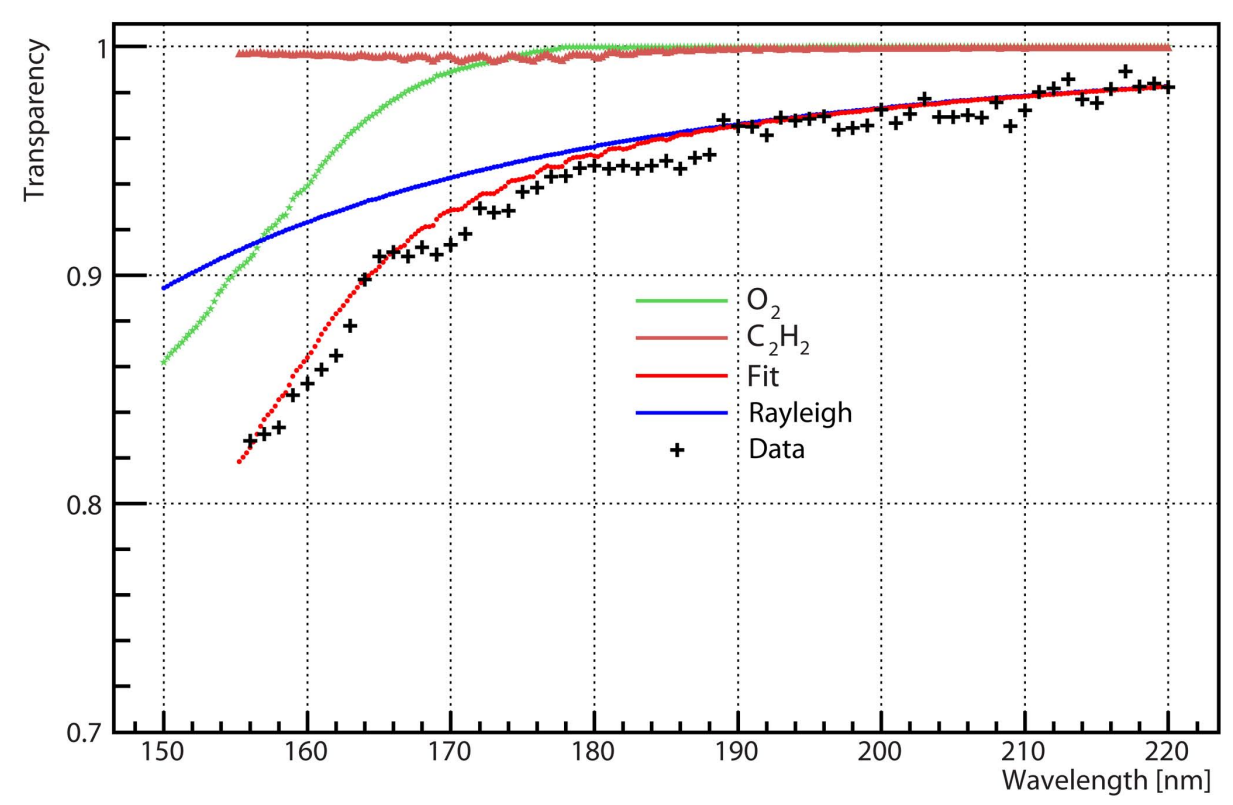

Figure 7. Typical example of a transparency curve for the $\mathrm{C}_{4} \mathrm{~F}_{10}$ of COMPASS RICH-1: the fitted transparency curve (in red) corresponds to contamination levels of $\mathrm{O}_{2}=2.3 \mathrm{ppm}, \mathrm{H}_{2} \mathrm{O}=0.0 \mathrm{ppm}, \mathrm{C}_{2} \mathrm{H}_{2}=$ $0.03 \mathrm{ppm}$.

\section{The mirrors and the CLAM system}

The Cherenkov photons generated inside RICH-1 are reflected by a $21 \mathrm{~m}^{2}$ mirror wall [11] (see figure 8) and focused on the photon detectors placed above and below the acceptance region. The mirror wall is divided in two spherical surfaces of $6.6 \mathrm{~m}$ radius and formed by 116 spherical mirror units: 68 regular hexagons with $522 \mathrm{~mm}$ long diagonal and 48 larger pentagons. The reflecting surface consists of an $80 \mathrm{~nm}$ thick $\mathrm{Al}$ layer deposited on a $7 \mathrm{~mm}$ thick borosilicate glass substrate and covered by a $30 \mathrm{~nm}$ tick $\mathrm{MgF}_{2}$ protective layer. 
A full characterization has been performed for each mirror before installation: with a radius of $6606 \pm 20 \mathrm{~mm}$, a $95 \%$ energy spot size of $1.65 \pm 0.45 \mathrm{~mm}$, a surface roughness r.m.s. of 1.26 $\pm 0.11 \mathrm{~nm}$ and a VUV reflectance of $83-87 \%$ in the range between 165 and $200 \mathrm{~nm}$ COMPASS mirrors exhibit very good optical properties. Their reflectance has remained stable in time after an initial degradation for the smallest wavelengths.

The mirrors are arranged in a mosaic-type composition with small clearance between them, corresponding to $4 \%$ of the total reflecting surface. They are suspended at the centre to the nodal points of the support structure via a joint which allows for small angular adjustments to be applied rotating two fine screws [11] (see figure 9)

The mechanical structure supporting the mirrors (see figure 10) has been designed to minimize the amount of material in the acceptance while preserving the accurate positioning of the mirrors. It has a net-like configuration, with nodal points laying on a sphere with a precision better than $1 \mathrm{~mm}$, obtained by assembling it on a dedicated mold manufactured for this operation (see figure 11).

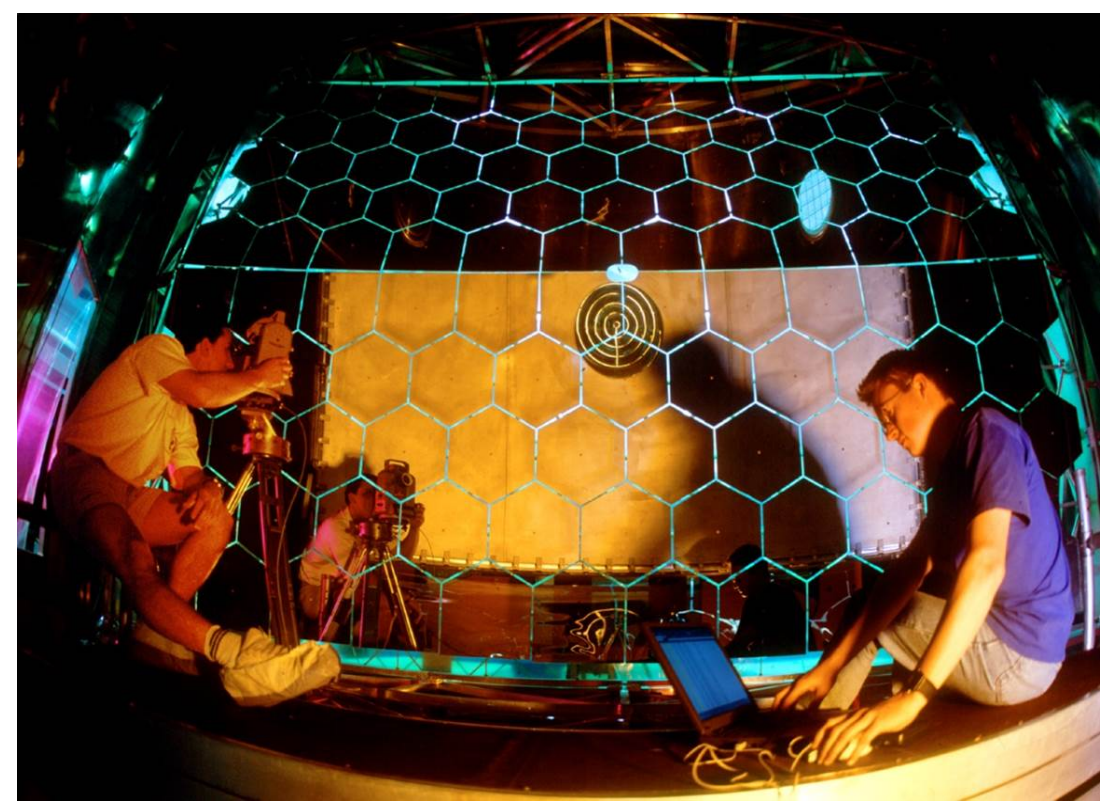

Figure 8. Picture of the mirror wall taken during the mirror alignment operation.
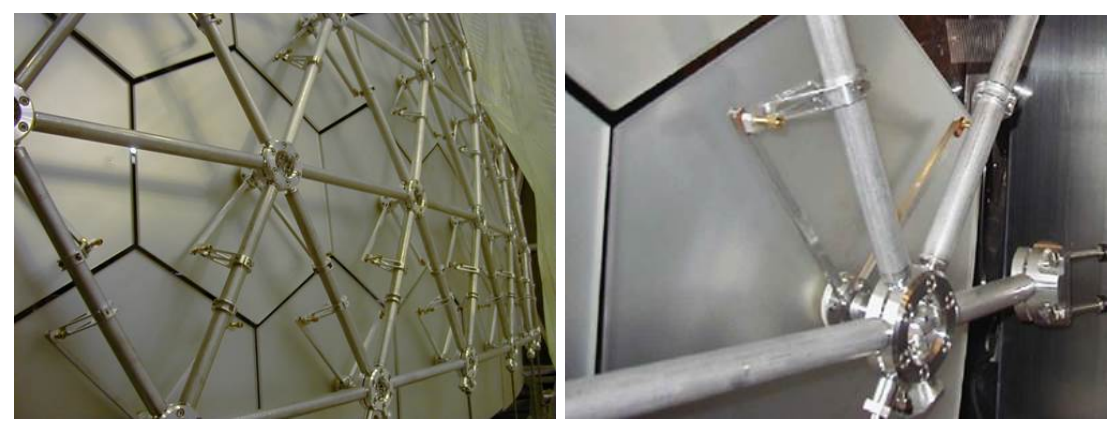

Figure 9. Picture of the back side of the mirrors and of the adjustment screws. 


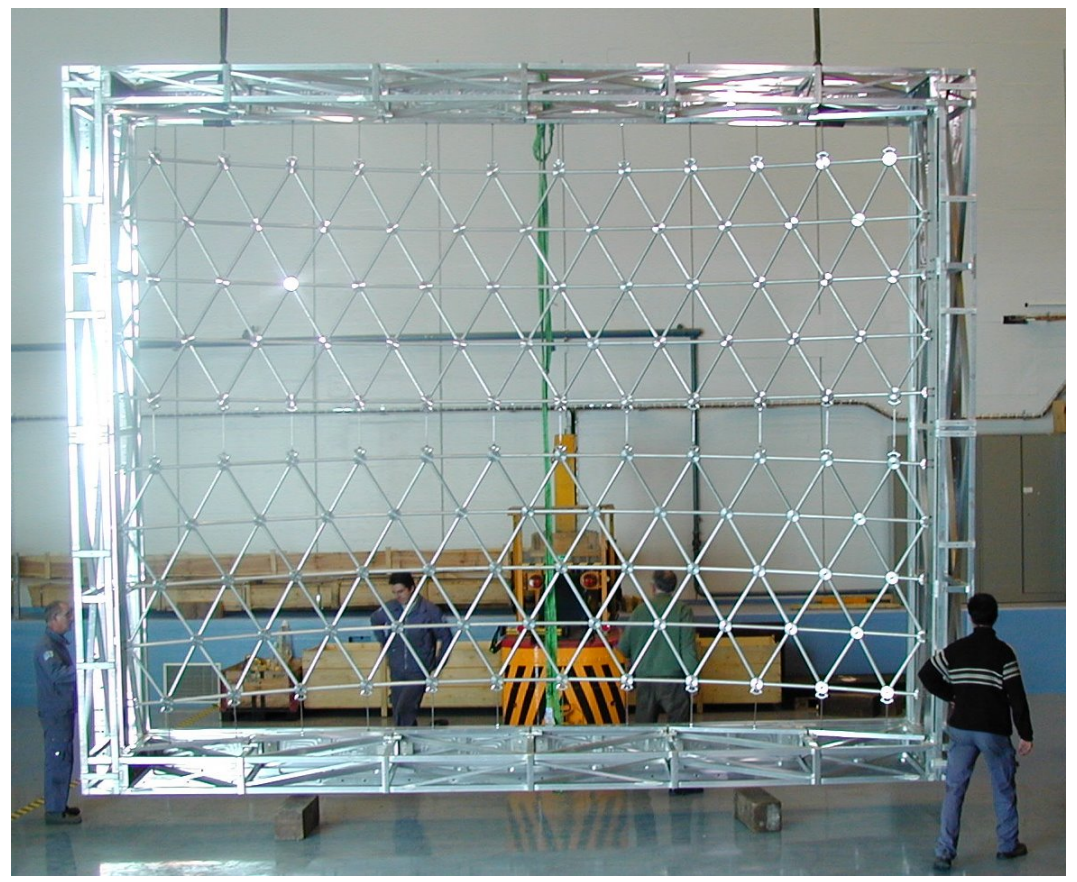

Figure 10. Picture of the mirror support structure: the nodal points are connected by light Al pipes.

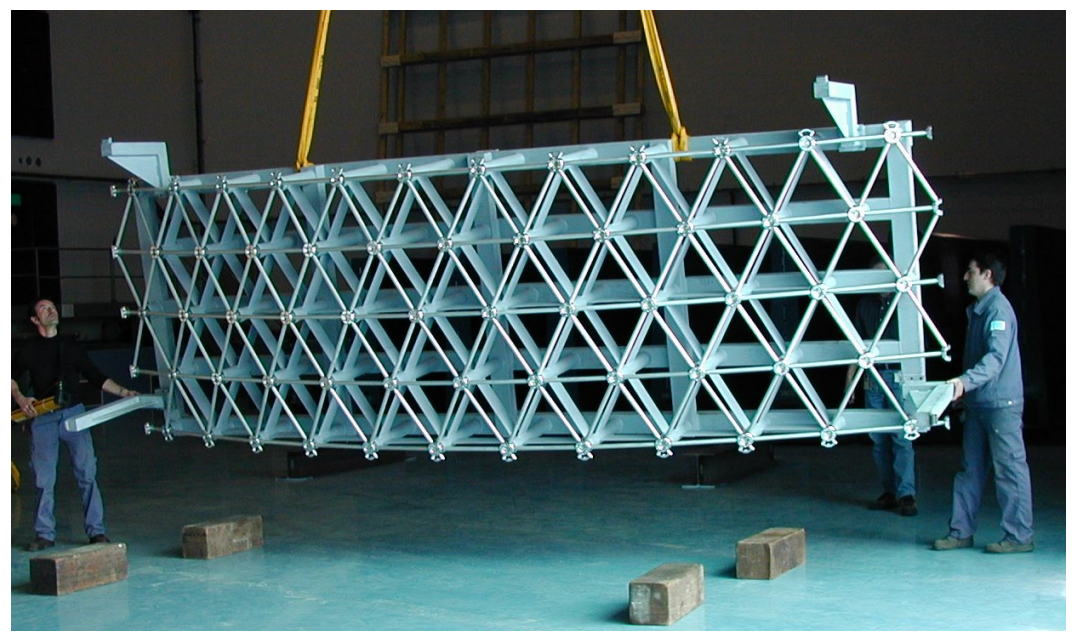

Figure 11. Picture of the half of the mirror support structure mounted on the dedicated mold.

As the mirrors have their centre of curvature located outside of the RICH, they have been individually aligned inside the RICH-1 vessel using the theodolite autoreflection method (see figure 8): the theodolite axis is oriented along the straight line joining its centre and the centre of the nominal mirror sphere (reference line); the mirror is than rotated until a laser beam along the reference line is reflected back on the same line. This procedure provides an accuracy of about $0.1 \mathrm{mrad}$.

The mirror alignment status has been checked after each COMPASS run: significant misalignment, up to $1 \mathrm{mrad}$, was detected for some mirrors at the end of the first run while no further large displacements have been detected later. 
The alignment check based on the theodolite autoreflection procedure implies long, risky and difficult operations: opening the RICH-1 vessel, mounting a scaffolding structure inside it and working close to the mirrors. In order to frequently monitor the mirror alignment during the data taking and to avoid risky operations an original on-line mirror alignment monitoring method [12,13] has been developed and implemented in 2007: four high resolution cameras, fixed on the corner of the vessel and facing the mirror wall (see figure 12) record the reflected image of a rectangular grid which is placed inside the vessel, close to the front wall. To increase the contrast of the images the grid is made of retro reflective material and equipped with optical targets while the scene illumination is provided for each camera by light sources located at the camera position.

The rectangular grid image is seen by a camera as a set of intersected conics: whenever neighboring mirrors have a relative misalignment, the conics lines appear to be broken and the shift of the line images provides the direction and amount of the relative misalignment. Individual calibration constants are needed for each mirror position to apply this procedure: they have been previously evaluated with accurate laboratory measurements.

The stability of the mirror orientation during the running period is checked by comparing images collected at different times: many slow continuous motions have been detected with maximum observed tilts of about $150 \mu \mathrm{rad}$.

By the use of photogrammetry for the complete characterization of each CLAM camera (with its optical system) and for the description of the geometrical position of each optical target inside the RICH it is possible to extract from the images the absolute position of the centre of curvature of each individual mirror [14].

The set of optical targets fixed on the mirror support frame is essential to determine the camera orientation and monitor its movements, allowing to disentangle their effects from the mirror generated ones.

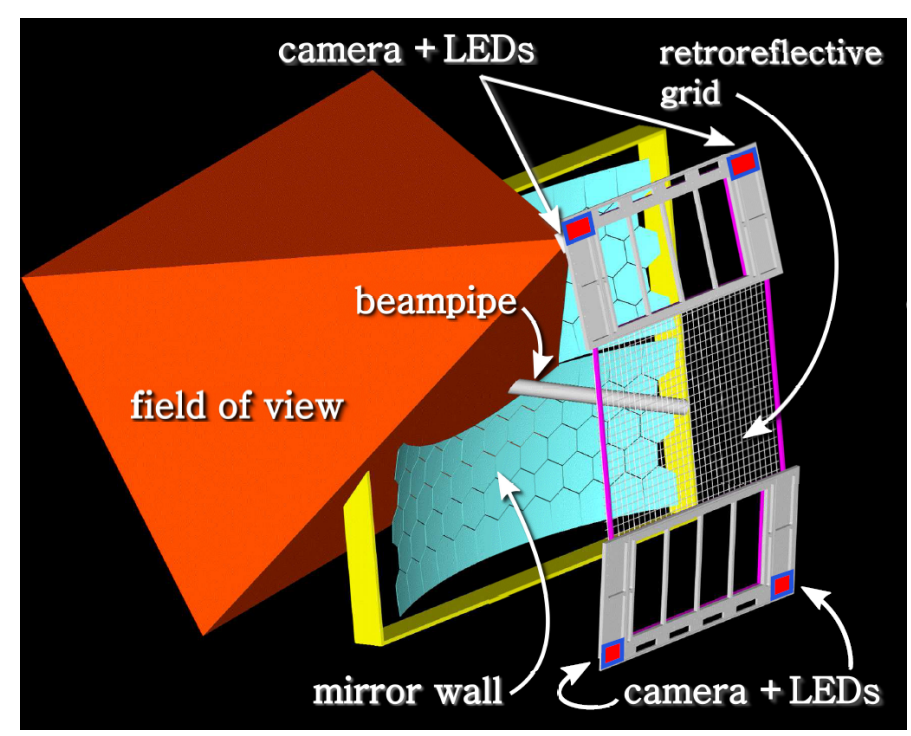

Figure 12. The CLAM hardware components; in red the field of view of one camera. 


\section{The MWPC-based photon detectors}

In its original version RICH-1 [4, 5] used as photon detectors eight MWPCs [15] (see figure 14) having $576 \times 1152 \mathrm{~mm}^{2}$ active area, each equipped with two square CsI-coated photocathodes (see figure 13), segmented in pads.

The principle of operation and the main parameters of this PDs [16] have been defined and optimized by the RD26 Collaboration: $2 \mathrm{~mm}$ for each anode-cathode gap, anode wires with diameter $=20 \mu \mathrm{m}$ and pitch $=4 \mathrm{~mm}$, cathode wires with diameter $=50 \mu \mathrm{m}$ and pitch $=2 \mathrm{~mm}$, cathode pads of $8 \times 8 \mathrm{~mm}^{2}$. Each photocathode has $72 \times 72$ pads which are individually read by the electronics, for a total of 82944 channels. Tight mechanical tolerances have been imposed in the construction phase to all components of the PDsand to their assembly (see figure 14), (50 $\mu \mathrm{m}$ for the anodecathode gaps, for instance), and great care has been put to all aspects of the construction of the MWPCs and the large quartz windows which separate the radiator gas from the pure methane used in the MWPCs (see figure 13).
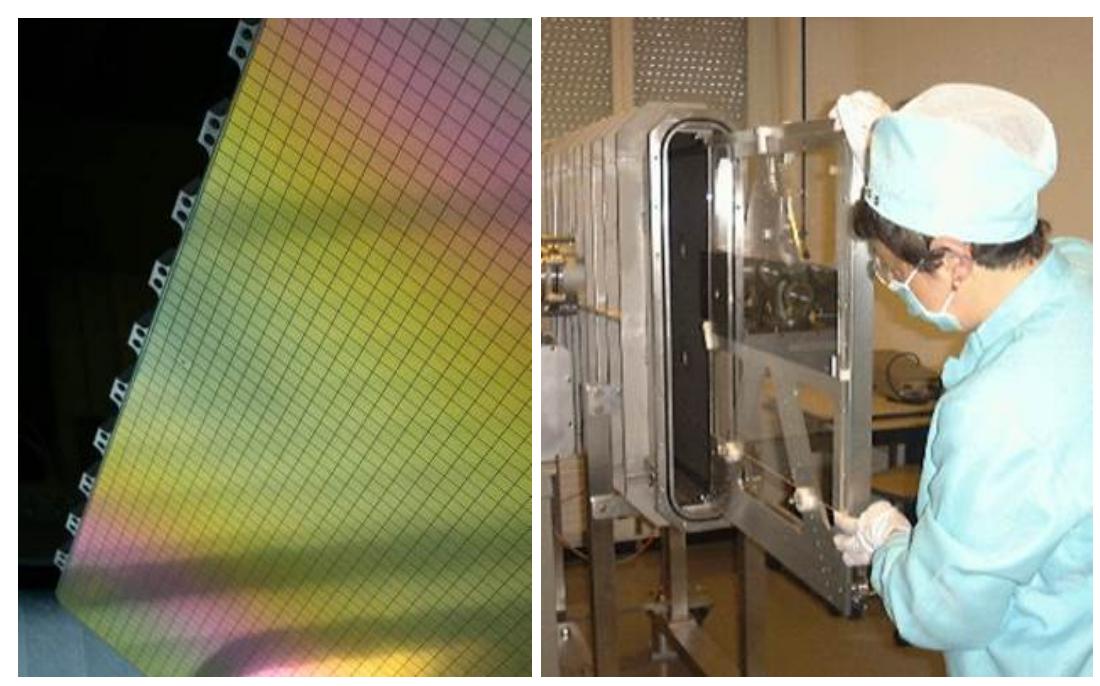

Figure 13. Picture of a CsI-coated photocathode and of a quartz window during the transparency measurement operation.

The CsI coating of the photocathodes (see figure 15) was performed at CERN, and dedicated tools and procedures were developed for the transport and handling of the photocathodes (see figure 16), in order to avoid exposure to air, in particular to water vapour, which would result in a degradation of the quantum efficiency. $[17,18]$ During all phases of the handling and storage they were kept in a controlled atmosphere with $\mathrm{O}_{2}$ level below $50 \mathrm{ppm}$ and flushed with clean, dry gas.

All MWPCs with CsI photocathodes have been tested in the laboratory to provide gains between $10^{5}$ and $10^{6}$, but they can be stably operated in the COMPASS experimental environment only at gains not exceeding $5 \times 10^{4}$ : for larger gains they exhibit occasional electrical instabilities with long ( $\sim 1$ day) recovery time, clearly related to the combined effect of the level of the applied voltage and the ionizing particle flux, as demonstrated by a series of dedicated studies and validation tests performed on these PDs at the CERN Gamma Irradiation Facility. 

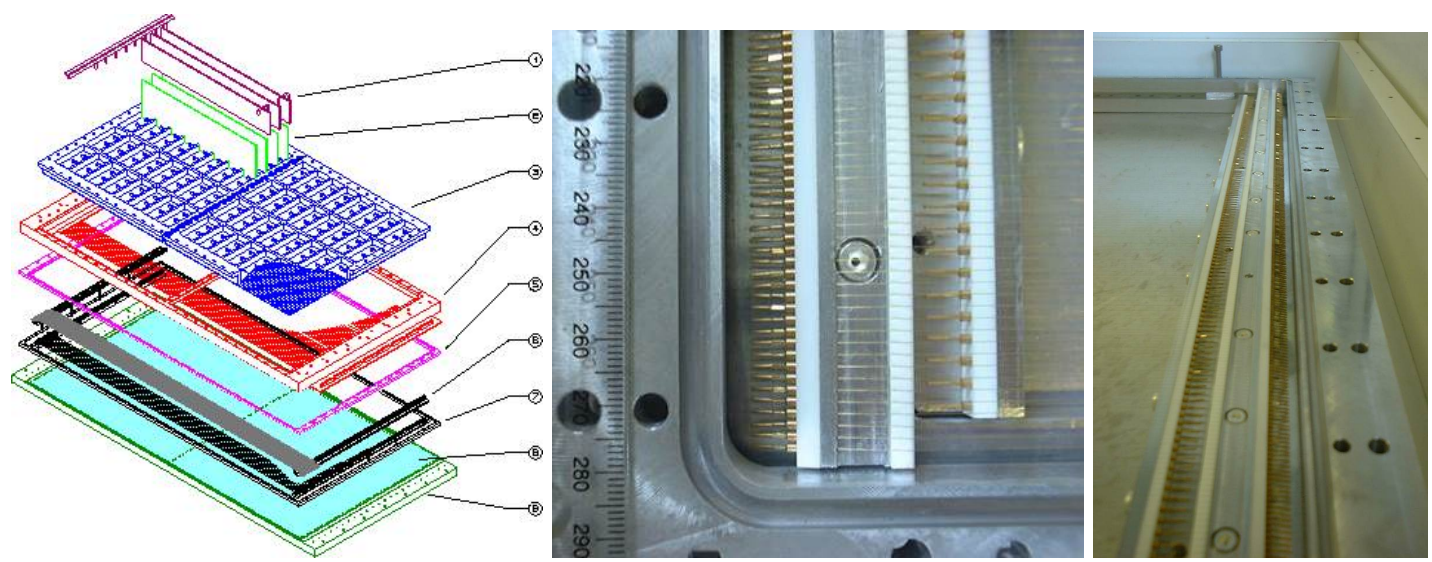

Figure 14. The structure and some details of RICH-1 MWPCs.
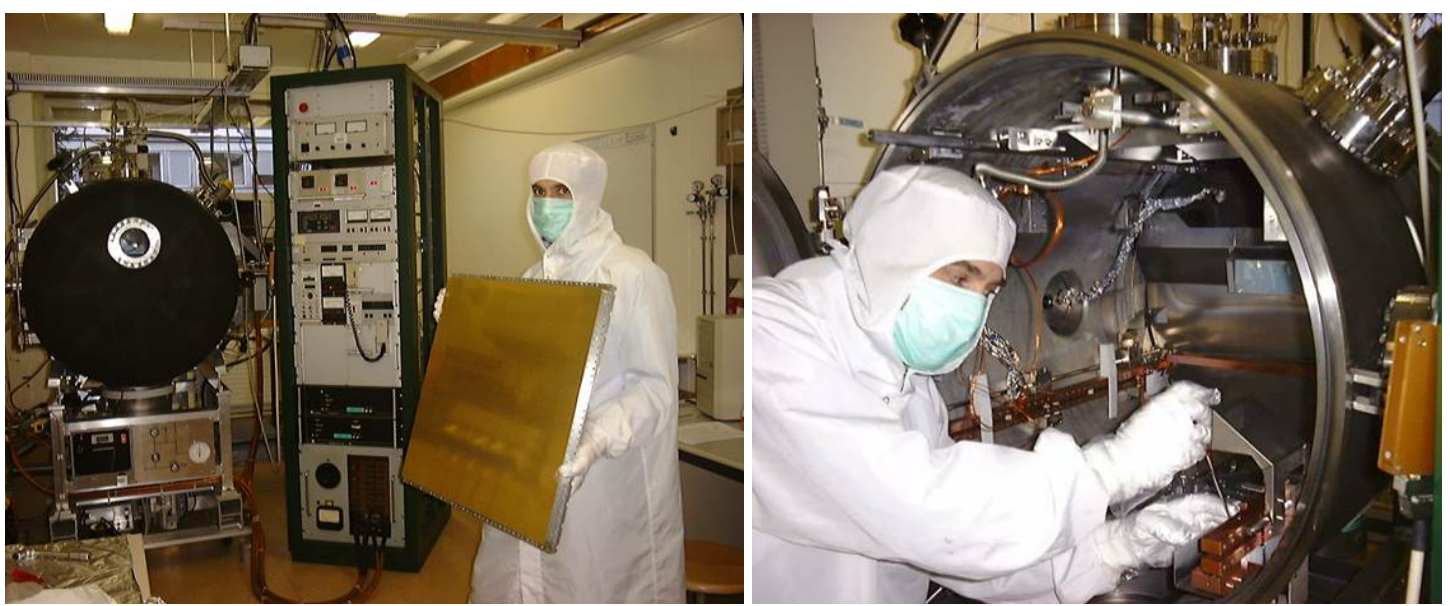

Figure 15. Pictures of the COMPASS photocathode and the CERN CsI evaporation plant.
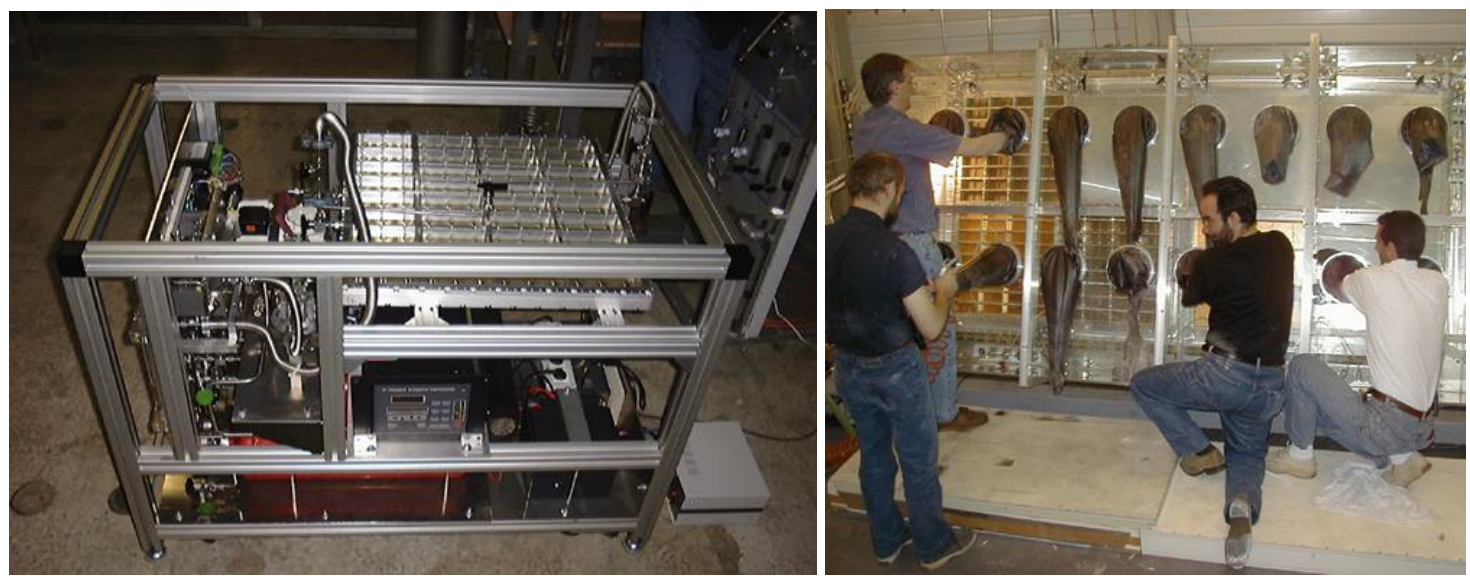

Figure 16. Pictures of the COMPASS photocathode transport system and of the the installation glove-box. 
The front-end electronics is directly plugged on the external side of the photocathode PCB and performs amplification and shaping of the signal; digitization takes place at the detector and the data are transmitted via optical fibres to the COMPASS DAQ system.

The readout $[19,20]$ in use before 2006 could provide a single photoelectron detection efficiency around $70 \%$ with an integration time of $0.6 \mu \mathrm{s}$, which was also acting as a detector memory and a $3.5 \mu$ s long baseline restoration time, generating a significant data acquisition dead-time.

For this reason the MWPCs with CsI photocathodes covering the outer region (75\% of the photon detection surface, corresponding to 62208 channels) have been equipped in 2006 with a new readout system [21] based on the APV25-S1 chip [22]: a 128-channel preamplifier and shaper ASIC whose output is sampled at a frequency of $40 \mathrm{MHz}$ and stored it in a 192 cell analog pipeline.

When an event is triggered, the cells to be read are multiplexed into a single differential output; two additional samples taken at the beginning and at the middle of the rising edge of the signal (300 and $150 \mathrm{~ns}$ before the maximum) are read to extract timing information. A 10-bit flash ADC and a FPGA are used to perform digitization and online zero suppression. The measured noise level is $\sim 680 e^{-}$to be compared with the average collected signal amplitude of $\sim 9000 e^{-}$.

An estimate of the CsI QE variation versus time has been obtained [23] by monitoring the mean number of detected photons per incoming charged pion in the COMPASS data for the period from 2006 to 2011.

Each year is characterized by different data taking conditions, including different triggers, targets and beams. To select samples as homogeneous as possible, in spite of different experimental conditions, the same cuts have been applied to the reconstructed particle trajectories.

Tracks identified as pions by means of the particle identification criteria described in [24], based on a likelihood analysis, have been selected: they have an associated Cherenkov ring provided by the standard patter recognition algorithm of COMPASS RICH-1 data analysis.

The amount and distribution of the background photons in the detectors is non-homogeneous and depends on the data taking conditions; variations in the the radiator gas characteristics, in the electronics settings and in the MWPC HV induce systematic effects which have been estimated to be small, apart from those due to a reduction by $20 \mathrm{~V}$ of the HV anodic bias of the MWPCs for the last two years. A correction (about $2 \%$ of the number of detected photons) has been evaluated and applied to compensate for the HV reduction.

The flux of ions to the photocathodes, integrated over the considered period, is smaller than $10 \mu \mathrm{C} / \mathrm{cm}^{2}$, corresponding to less than $1 \%$ of the flux reported in literature $[17,18]$ to cause significant $\mathrm{QE}$ reduction.

For each of the twelve CsI photocathodes a set of tracks has been selected requiring that the associated Cherenkov ring is completely contained in the photocathode: the selected tracks are characterized by low momenta (with mean values between 3 and $4 \mathrm{GeV} / c$ ) and therefore the Cherenkov angle distributions are statistically poor in the region of the largest values of the Cherenkov angle, as can be seen in figure 17, where the distribution of the pion momenta for rings fully contained in one of the cathodes (cathode n. 5) is presented. In the same figure the Cherenkov angles distribution for the associated rings is shown.

For each ring the value of the Cherenkov angle $\theta_{C h}$ and the number of detected photons $\mathrm{N}_{p h}$ are obtained. The data are grouped in $1 \mathrm{mrad}$ wide $\theta_{C h}$ bins and for each bin the mean value of the $\mathrm{N}_{p h}$ distribution is computed: in figure 18 the mean value of the $\mathrm{N}_{p h}$ for one year and one 

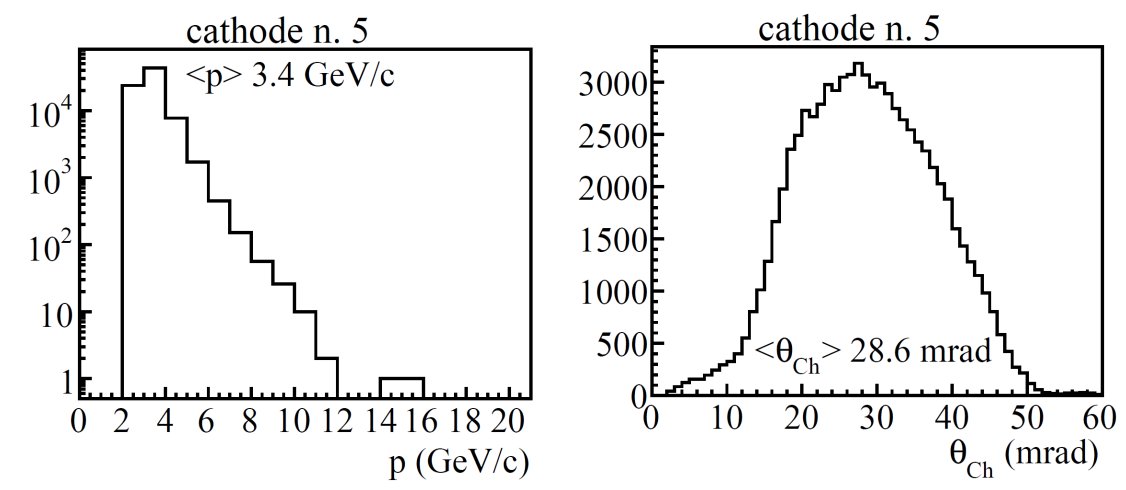

Figure 17. Momentum distribution and Cherenkov angle distribution of the pions with a ring fully contained in one of the photocathodes (cathode n. 5).
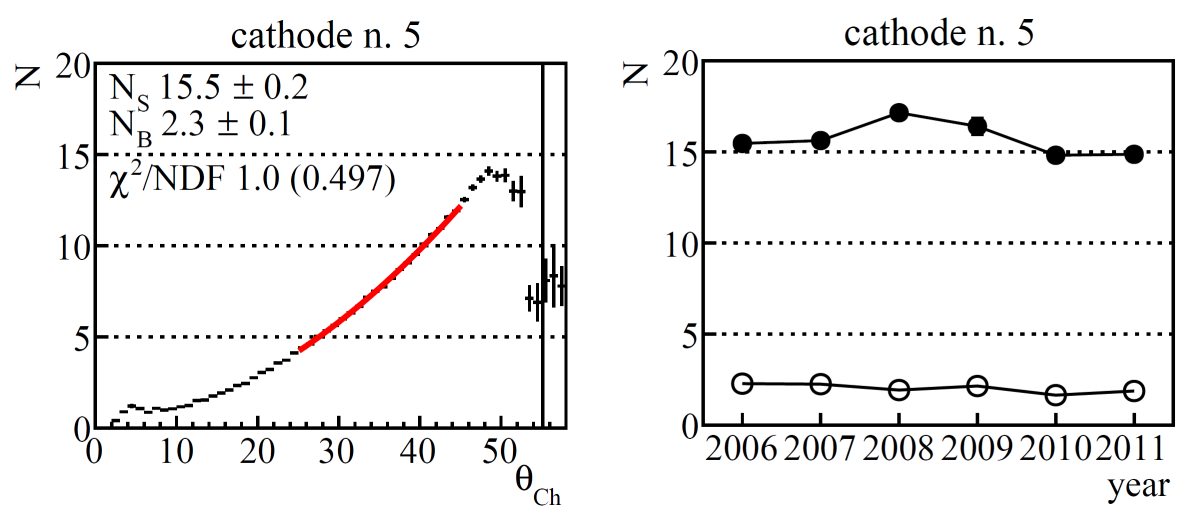

Figure 18. Number of detected Cherenkov photons versus Cherenkov angle and variation over six years of the extrapolated number of detected Cherenkov photons for $\theta_{C h}=55.2 \mathrm{mrad}$ for the pions with a ring fully contained in one of the photocathodes (cathode n. 5).

cathode (year 2006 and cathode n. 5) is plotted for each $\theta_{C h}$ bin. The detected photons $\mathrm{N}_{p h}$ are expected to include a background contribution, $\mathrm{N}_{B}$, with a uniform distribution, corresponding to a linear dependence on $\theta_{C h}$ and a signal component, $\mathrm{N}_{S}$ following the Frank and Tamm distribution, implying a $\sin ^{2}\left(\theta_{C h}\right)$ dependence. The fit to the $\mathrm{N}_{p h}$ distribution is performed with the function:

$$
\mathrm{N}_{p h}\left(\theta_{C h}\right)=p_{0} \cdot \sin ^{2}\left(\theta_{C h}\right)+p_{1} \cdot \theta_{C h}
$$

where $p_{0}$ and $p_{1}$ are free parameters; the fit allows to separately determine the signal and the background contributions. It is applied to the distribution of $\mathrm{N}_{p h}\left(\theta_{C h}\right)$ in the central range of $\theta_{C h}$ values, as shown by the continuous red line in figure 18 , because for the large $\theta_{C h}$ bins the statistics is poor and for small $\theta_{C h}$ bins the measured distribution is not following the Frank and Tamm law, due to the missing detection of Cherenkov rings with no photons.

The number of detected signal photons is expected to follow Poisson statistics since the number of emitted Cherenkov photons does it, if the effects of photon signal overlap and other sources of distortions are small; the mean value of the measured distribution is however biased because the zero-photon rings are not detected: this effect is very relevant for the small $\theta_{C h}$ bins; after applying 
a correction for this effect the average number of detected signal photons decreases and approaches zero at small $\theta_{C h}$ as expected; this effect becomes negligible for $\theta_{C h}>25 \mathrm{mrad}$.

In general the fits are good, for all years and all cathodes, with one exception, corresponding to a cathode affected by electrical instabilities and exhibiting lower efficiency: this cathode has been excluded from the analysis. From the study of the $\chi^{2}$ distribution of the fits an estimate of the systematic uncertainty $\sigma_{\text {syst }}=0.7 \sigma_{\text {stat }}$ has been obtained. The dependence of the results on the $\theta_{C h}$ range used in the fit can be neglected.

In order to compare data from different years, with different running conditions, the background is subtracted and the fitted signal distributions are evaluated at a fixed reference value of $\theta_{C h}: \theta_{C h}^{\text {ref }}=55.2 \mathrm{mrad}$, corresponding to the Cherenkov angle at saturation $\theta_{C h}^{\max }$ in 2006 data; the different values of the radiator gas refractive index (mainly due to slightly different nitrogen contamination) result in different $\theta_{C h}^{\max }$ in the range from $55.2 \mathrm{mrad}$ to $55.4 \mathrm{mrad}$ in different years.

The values of $\mathrm{N}_{B}\left(\theta_{C h}^{\text {ref }}\right)$ has been evaluated for each of the 11 photocathodes [23] and for each year: an example of the observed variation is presented in figure 18. The error bars represent the statistical plus the systematic contribution which include a set of effects (temperature and pressure variations, electronic threshold setting, etc.) none of which contributes more than $1 \%$ of the measured number of detected photons.

In figure 19 the mean number of photons per ring at saturation, averaging the information from the 11 cathodes is presented as function of the year. The error bars not only include the evaluated systematic error contribution but also an enlargement factor obtained requiring the fit of a first order polynomial to provide a probability value of 0.5 .

No evidence for a substantial decrease of the CsI QE is observed, and the points are consistent (33.4\% CL) with a constant value, represented in the plot by the dashed line. The first order polynomial fit provides a slope of -0.28 photons/year, corresponding to an estimated decrease rate of the $\mathrm{QE}$ of $2.3 \%$ per year.

It can thus be said that the CsI photocathodes used by COMPASS RICH-1 are robust and preserve their QE over several years of operation with at most a moderate decrease.

\section{The MAPMT-based photon detectors, the readout and DAQ}

In 2006, to overcome the problem of low PID efficiency for very forward particles, to provide higher rate capability and to get rid of the large uncorrelated background, the MWPCs with CsI photocathodes in the central region (25\% of the detector surface) have been replaced by a fast detection system [25-30] based on MAPMTs coupled to individual fused silica lens telescopes and read out via sensitive front-end digital electronics and high resolution TDCs.

Four panels of 144 MAPMTs, (Hamamatsu R7600-03-M16 with 16 channels and a UV extended glass window), equipped with custom voltage dividers have been installed in place of the four central CsI coated photocathodes (see figure 20).

A total of 612 MAPMTs have been submitted to a complete quality control protocol [25, 31] including visual inspection, measurements of dark currents and of gain at 5 different voltages. The typical gain is about $10^{7}$ at $900 \mathrm{~V}$, with excellent uniformity; no gain reduction is observed up to a single photoelectron rate of at least $5 \mathrm{MHz}$ per channel. 


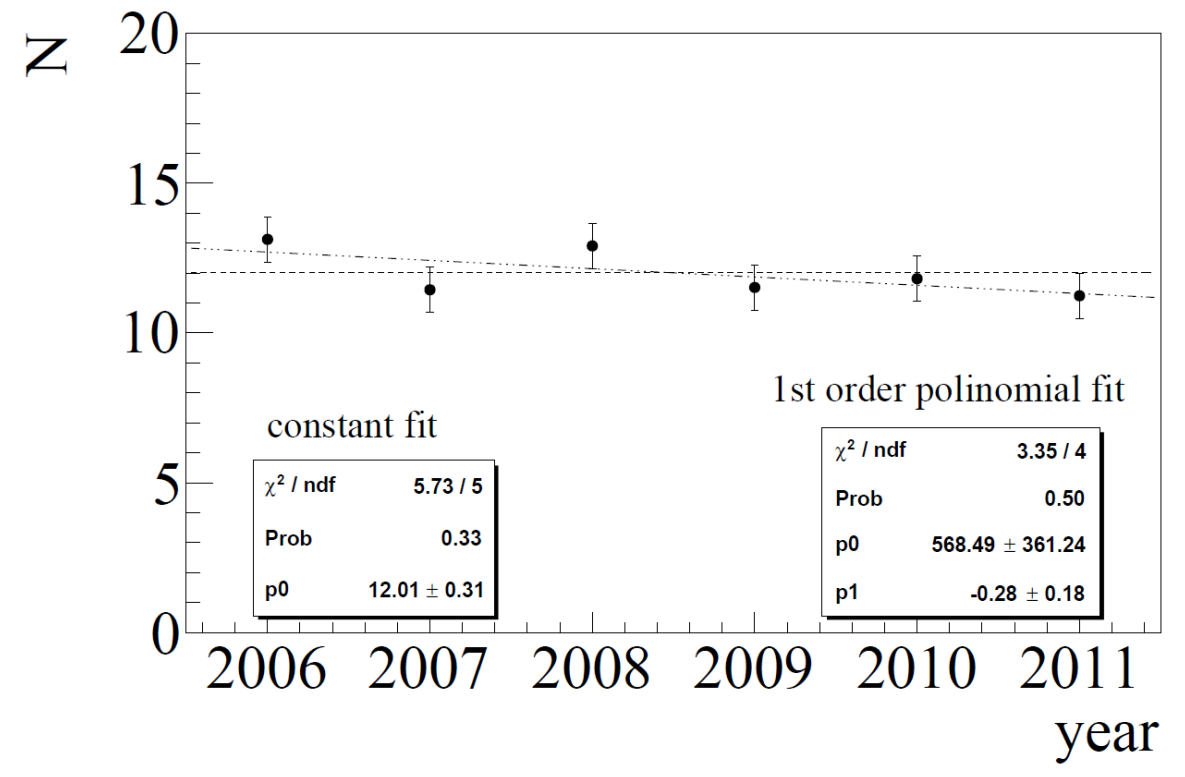

Figure 19. Mean number of detected signal photons for $\theta_{C h}=55.2 \mathrm{mrad}$ as function of the year averaged over 11 photocathodes. Results from a best fit procedure with a constant and a first order polynomial function are shown.

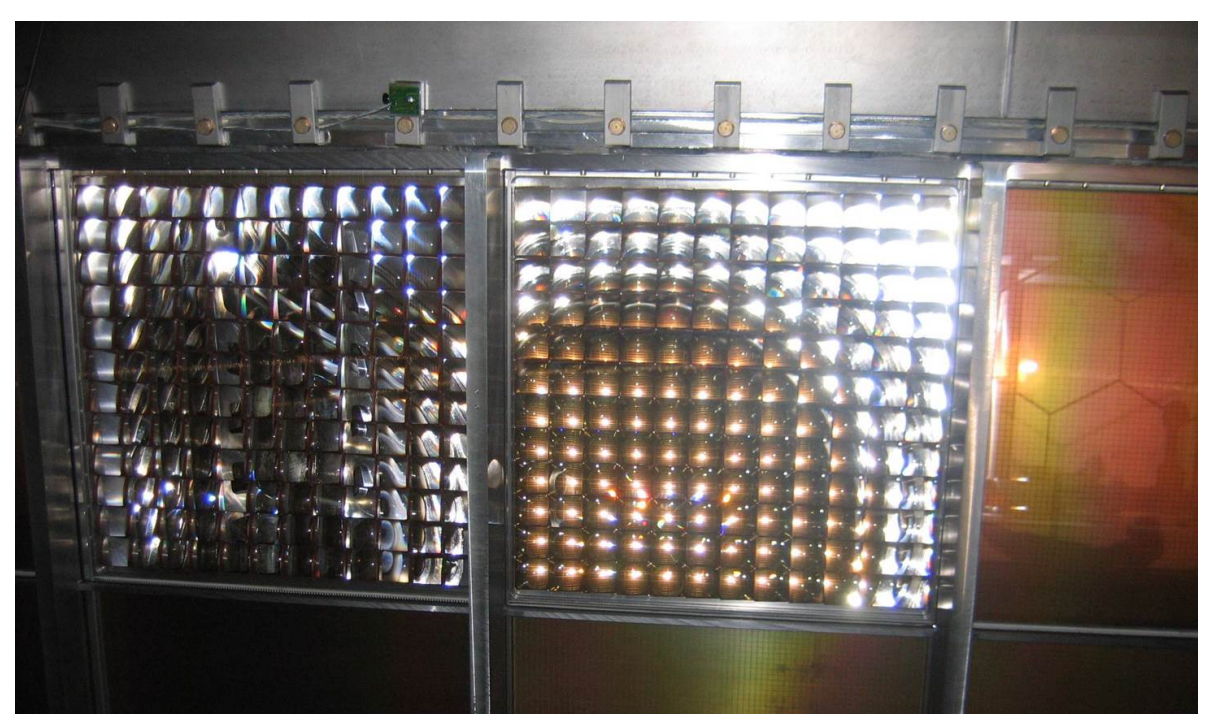

Figure 20. Two panels with lens telescopes and MAPMTs seen from inside of the RICH-1 vessel.

Individual soft iron boxes have been installed to screen the magnetic field of about 200 Gauss present at the PDs location.

In front of the MAPMTs a light concentration system transmits photons with wavelength in the range from 200 to $700 \mathrm{~nm}$ with a wide angular acceptance $\left(100 \%\right.$ up to $8.3^{\circ}, 50 \%$ at $\left.9.3^{\circ}\right)$, provides a large image reduction (a factor 7.3 in area) with minimal distortion, and complies with the space limitations at the detector (11.5 cm total length). It consists of individual optical telescopes [25, 32] 

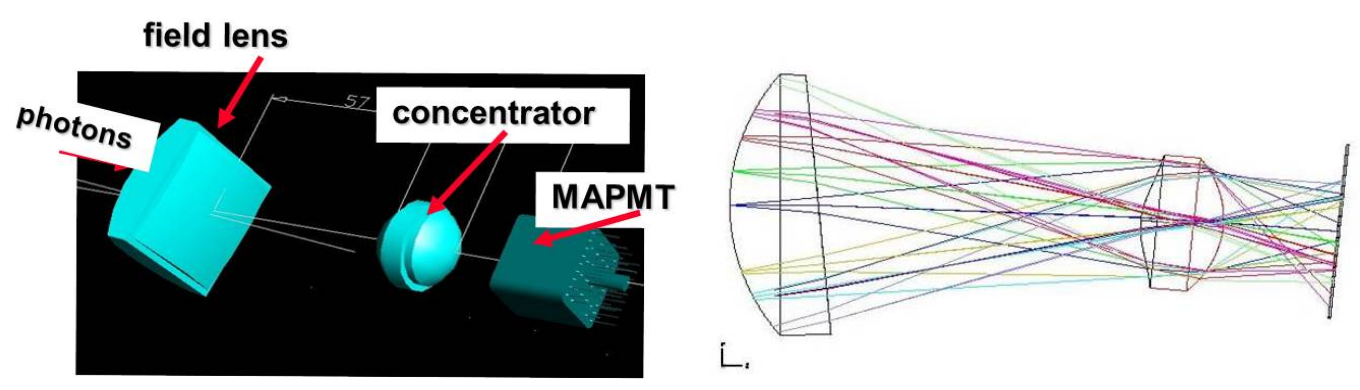

Figure 21. Artistic view and scheme of the individual fused silica lens telescope.

for each MAPMT, made of two fused silica lenses (see figure 21): a plano-convex field lens, placed in the focal plane of the mirrors and a biconvex condenser lens with one aspherical surface, providing the large demagnification with reduced image distortions. The image is projected to the plane of the MAPMT photo-cathode with a total spot size r.m.s. of $1 \mathrm{~mm}$, to be compared to the $4.5 \mathrm{~mm}$ pitch of the MAPMT pixels.

The fused silica lenses are made by grinding and polishing procedure with tight tolerances for surface quality and shape, and are coated with a $\mathrm{MgF}_{2}$ antireflection layer which provides an increase of about $8 \%$ in the number of the collected photons. Each lens and each telescope have been tested [25, 33] in a custom setup employing the Hartmann method [34, 35] to provide individual characterization of wavefront distortions with respect to ideal optics; the final image displacement introduced by optics imperfections is below $50 \mu \mathrm{m}$ for $70 \%$ of the telescopes, and in all cases below $150 \mu \mathrm{m}$. A careful mechanical design of the lens support frames (see figure 22) allowed to reduce the dead areas below $2 \%$ of the surface.
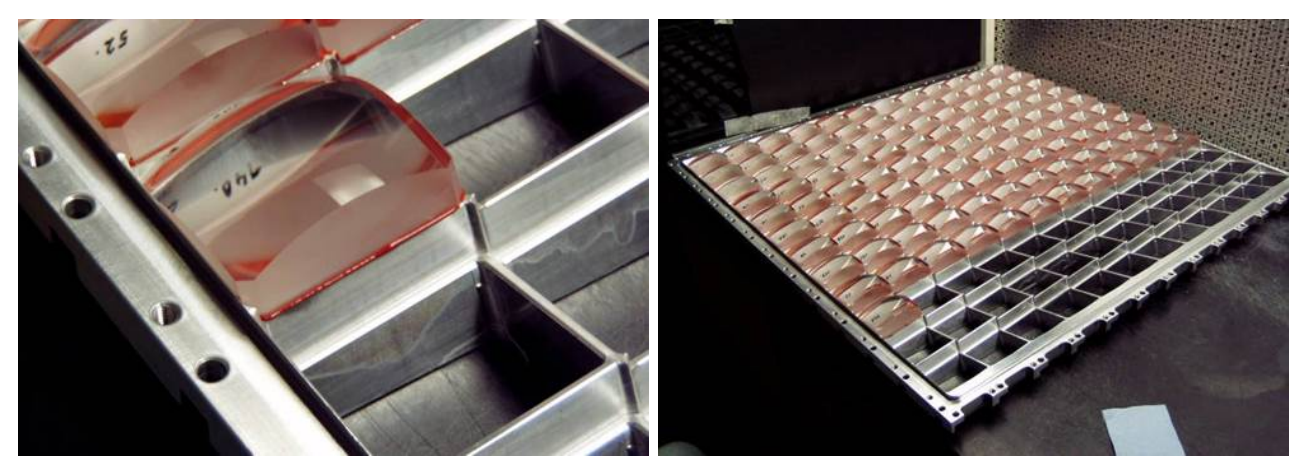

Figure 22. Picture of a lens and of a lens panel during the lens glueing operation.

The signals from the MAPMTs are read by a fast digital electronics system [36, 37] based on the 8 channels CMAD [38] preamplifier-discriminator, developed for COMPASS RICH-1 as an upgraded version in CMOS technology of the MAD4 [39] front-end chip. The CMAD has a small noise level ( $\sim 1 \mathrm{fC}$ ), the possibility to set individual channel thresholds, a good time resolution and high rate capability: it provides full efficiency up to an input rate of $5 \mathrm{MHz}$ per channel. The design of the front-end boards and the optimization of the thresholds setting allows to completely suppress the MAPMTs cross talk signals while keeping the single photoelectron detection efficiency at a $\sim 95 \%$ level. 
The good MAPMT time resolution is fully exploited with the help of digital cards, called DREISAM, housing the dead-time free F1 TDC [40], which has a time resolution of $\sim 110 \mathrm{ps}$ and can stably operate up to $10 \mathrm{MHz}$ per channel input rate and $100 \mathrm{kHz}$ trigger rate.

All the electronics components of the RICH-1 readout system are directly mounted on the detector and form a very compact setup. Each PCB is coupled to a copper plate providing both efficient electromagnetic shielding and good cooling power: thermalized water circulates in underpressure condition in thin copper pipes brazed onto the copper plates [36]. The stability and uniformity of the water cooling system has been achieved after several improvements of the distribution system and of the operation and maintenance protocols.

Data from the front-end cards are transferred via optical links to a set of CATCH readoutdriver modules which concentrate the data and send them via S-LINK transmitter and optical fibre to the COMPASS DAQ system.

\section{PID performance}

A dedicated reconstruction and analysis package, called RICHONE [24], has been developed to perform data reduction, pattern recognition and hadron identification in COMPASS; it is also used to perform several accessory tasks, including tuning and calibrations and to study the RICH-1 detector performance. Its software is part of CORAL, the COMPASS reconstruction and analysis package [3].

For each event the RICH-1 data are decoded, the MAPMT hits are selected on the basis of the time information and the MWPCs hits are selected on the base of the time and amplitude information and clustered; all accepted particles, namely tracks within the RICH angular acceptance and having a momentum between 1.8 and $180 \mathrm{GeV} / c$, are then correlated to the RICH reconstructed coordinates.

For each particle a wide fiducial area of the photon detectors is defined, where its Cherenkov photons could have arrived, which can sometimes cover both part of the MAPMT and part of the MWPC or part of the upper and part of the lower photon detector set; for each reconstructed hit inside the fiducial area an algorithm is used to compute the Cherenkov polar and azimuthal angles in the particle reference frame: this are the reconstructed photon parameters used in the analysis. Corrections for the individual mirror parameters and the MAPMT optical system crossed by the photon are included.

The reconstructed photons are relative to the considered particle and the reconstruction algorithm is repeated for each particle in the event.

The value of an extended likelihood function is computed for five mass hypotheses $(e, \mu, \pi, K, p)$ and one for the absence of signal hypothesis: the maximum is assumed to correspond to the best hypothesis. The identification purity can be improved by requiring the ratio of best to second best hypothesis likelihood to be larger than a fixed value.

In figure 23 the measured ring Cherenkov angle $\theta_{C h}$ as a function of the particle momentum is shown. The $e, \pi, K$ and $p$ band are clearly separated. In figure 24 another relationship between the Cherenkov angle and the particle momentum is shown, which emphasises the small momentum region: $\theta_{C h}^{2}$ as a function of $1 / p_{2}$. In the small angle approximation, the relationship between these two variables is linear, with a slope proportional to the squared mass of the corresponding particle. 


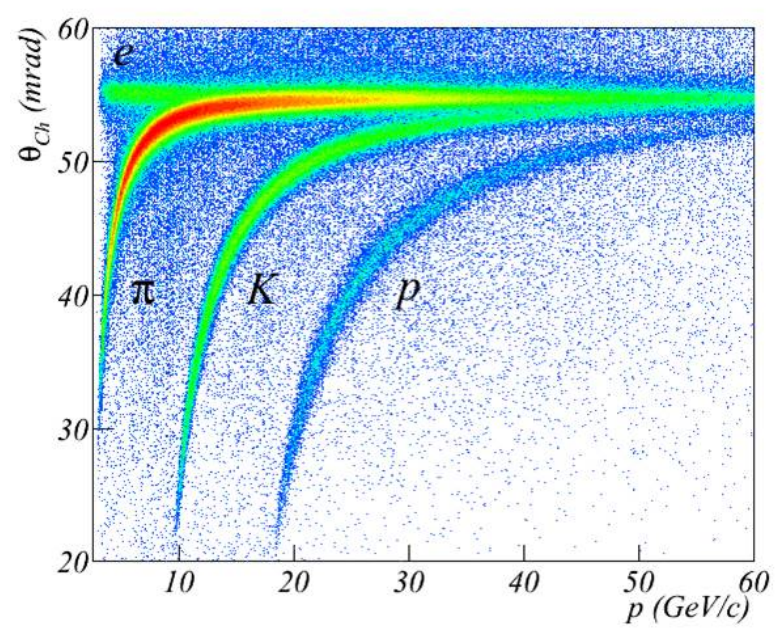

Figure 23. The measured ring Cherenkov angle versus particle momentum $p$.

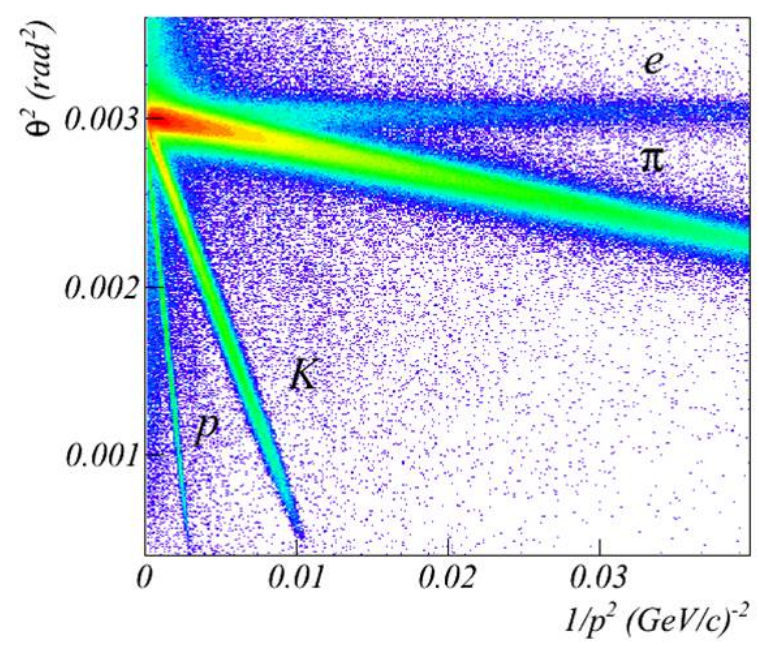

Figure 24. The measured ring Cherenkov angle squared versus $1 / p$.

For an accurate likelihood evaluation the average refractive index of the radiator gas needs to be know at about $10^{-6}$ level for both detector types: it is experimentally determined from the RICH-1 data at fixed time intervals and evolved during the intermediate time periods according to the measured changes in the radiator temperature and pressure.

In parallel, ring pattern recognition is performed by the RICHONE package; this is essential for a detailed study of the RICH response and for the fine tuning and optimization of both the RICH detector hardware and the data analysis program.

The number of detected photons per ring as function of the Cherenkov angle for rings fully contained in the MAPMT part of the photon detectors is shown in figure 25: for particles with $\beta=1$ the average number of detected photons is 56 . 


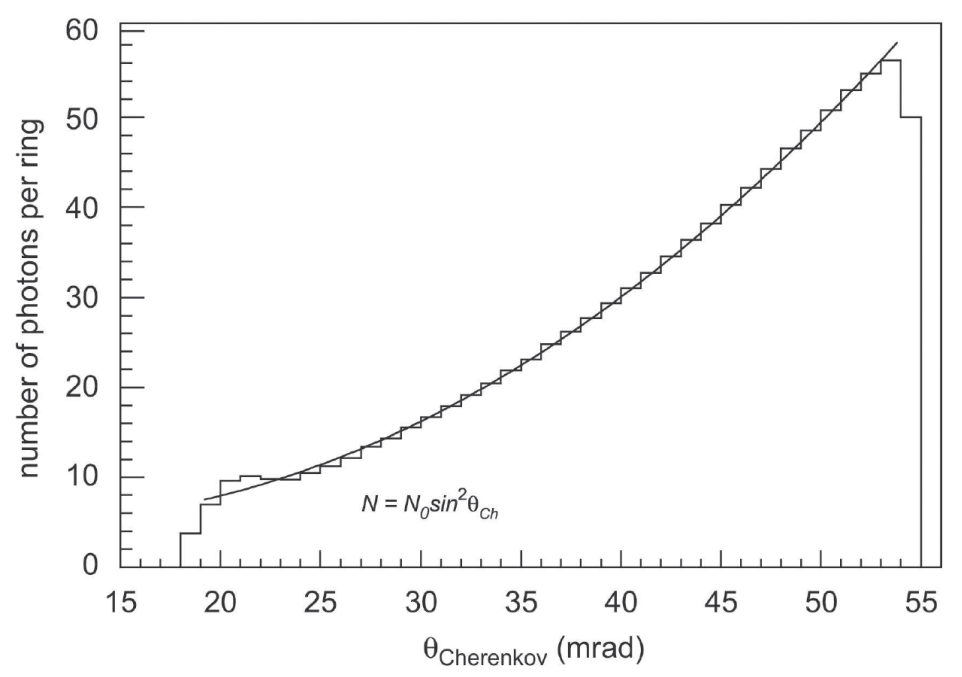

Figure 25. Number of photons per ring as function of the Cherenkov angle.

The measured single photon resolution in the central region is $2.0 \mathrm{mrad}$ and the global resolution on the measured Cherenkov angle [41] is less than $0.3 \mathrm{mrad}$ for particles at saturation, in agreement with the single photon resolution value scaled by the square root of the number of photons: this confirms that the background contribution is minimal, thanks to the excellent time resolution of the MAPMTs.

In the central region, where the ring images produced by high momentum particles are formed, the $2 \sigma \pi$-K separation defined according to the recipe of ref. [42] is up to $55 \mathrm{GeV} / c$.

Making use of a sample of kinematically reconstructed $K_{S}^{0}(\phi(1020))$ mesons, the efficiency for $\pi(K)$ identification has been evaluated as function of particle momenta: figure 26 (27) shows the efficiency and the misidentification probability for $\pi(K)$ for the three most commonly used cuts on the ratio of the best to the second best hypothesis likelihood. For particle momenta below $30 \mathrm{GeV} / c$ the efficiencies are larger than $90 \%$ and the misidentification probabilities smaller than $1 \%$; for higher momenta the cut on the likelihood ratio can be tuned to optimize efficiencies or sample purities.

The purity of identified $K$ samples depends on the physics channel under consideration: figure 28 shows it for standard COMPASS deep inelastic scattering events. In the momentum range below $30 \mathrm{GeV} / c$ the purity is always above $80 \%$, for all cuts on the likelihood ratio.

The identification of hadrons with momenta below the Cherenkov threshold is performed in a very natural way by using the likelihood for the absence of signal hypothesis; thanks to the low background level it turns out to be very effective too: $K$ samples with momenta below $9 \mathrm{GeV} / c$ from the decay of $\phi(1020)$ show identification efficiencies larger than $90 \%$.

\section{Perspectives and conclusions}

In order to cope with the challenging requests posed by the future physics program of COMPASS a set of new generation, high performing photon detectors with an active area of $576 \times 576 \mathrm{~mm}^{2}$ 

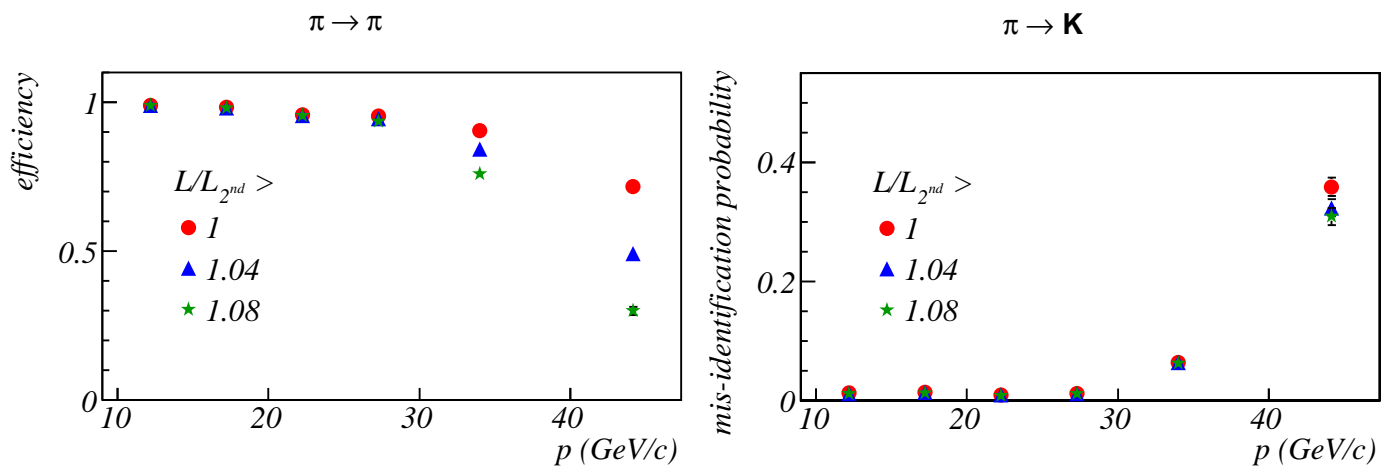

Figure 26. Efficiency and misidentification probability for $\pi$ as function of momentum.
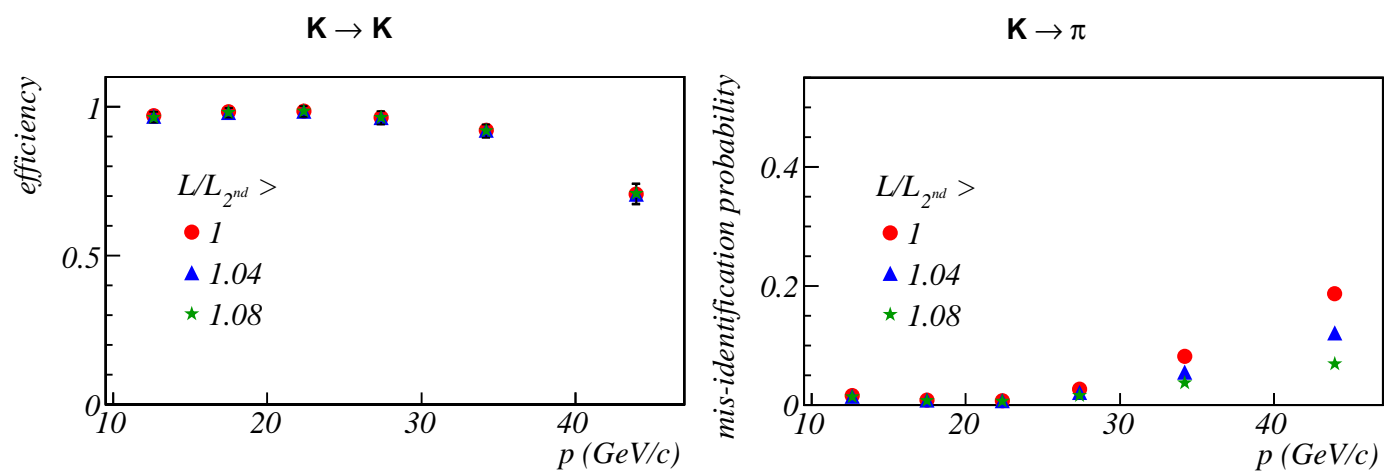

Figure 27. Efficiency and misidentification probability for $K$ as function of momentum.

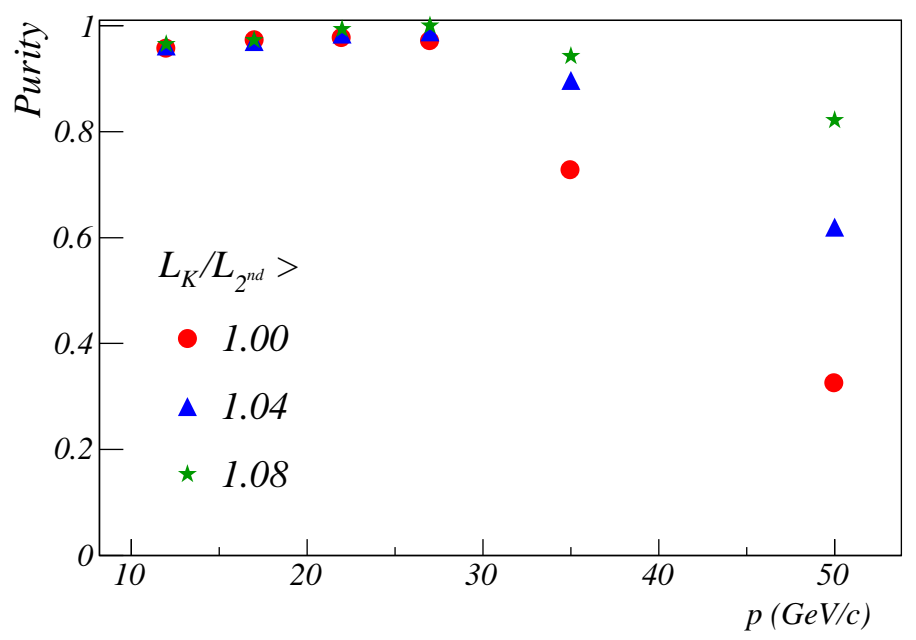

Figure 28. Purity of $K$ samples as function of momentum. 
will be installed. The characteristics of the new detectors are:

- a small signal formation time to provide a time resolution $\leq 10 \mathrm{~ns}$,

- a closed geometry to avoid photon feedback,

- a large gain $\left(\geq 10^{5}\right)$ to guarantee single photoelectron detection efficiency and avoid critical dependence on the front-end electronics thresholds,

- a reduced ion back-flow to the CsI photocathode $(\leq$ few $\%)$ to guarantee stability and avoid aging problems,

- a good stability of the response both in time and under different irradiation conditions to guarantee high and constant efficiency.

A dedicated R\&D program [43-48] aimed to develop novel Photon Detectors (PDs) with the above mentioned characteristics was started six years ago in view of the upgrade of the COMPASS RICH-1 detector.

Micro-Pattern Gaseous Detectors (MPGDs) have been chosen as base elements for the new detectors, since they meet the fundamental requirements and Gas Electron Multipliers (GEMs) [49] had been successfully used as PD elements in the Hadron Blind Detector [50, 51] of the PHENIX Experiment at RHIC, although not as detectors of single photons.

A more robust solution with several advantages is provided by the use of THGEMs [52-55]. They are gaseous electron multipliers derived from the GEM design, scaling the geometrical parameters and changing the production technology: standard Printed Circuit Boards (PCBs) are used instead of the $\mathrm{Cu}$-coated polyimide foils and the holes are obtained by drilling. They can be industrially manufactured using standard PCB drilling and etching techniques.

Chambers hosting multilayer THGEM arrangements with CsI coating on the top of the first THGEM have been built and operated in the laboratory and at the CERN Test Beam lines [57]: they provide effective gain in the range of $10^{5}-10^{6}$ and time resolution below $10 \mathrm{~ns}$.

Various alternative solutions have been studied and the best results in terms of performance and stability have been obtained using chambers built combining Micromegas [56] and THGEM technologies. This hybrid configuration has been chosen for the future RICH-1 Photon Detectors.

A full-size prototype is presently under construction and the implementation of the new detectors (see figure 29) on COMPASS RICH-1 is foreseen for 2016.

In conclusion:

- The RICH-1 detector of the COMPASS Experiment at CERN provides outstanding PID performance: hadron identification between 3 and $55 \mathrm{GeV} / c$ at high beam and trigger rates, over a wide angular acceptance.

- The experience of building and operating it for more than 10 years has been exciting and successful. Original solutions had to be found for several challenging problems in order to reach high and stable performance.

- For the physics runs starting in 2016 COMPASS RICH-1 will be equipped with new MPGDbased photon detectors, which have been developed by a dedicated R\&D program. 


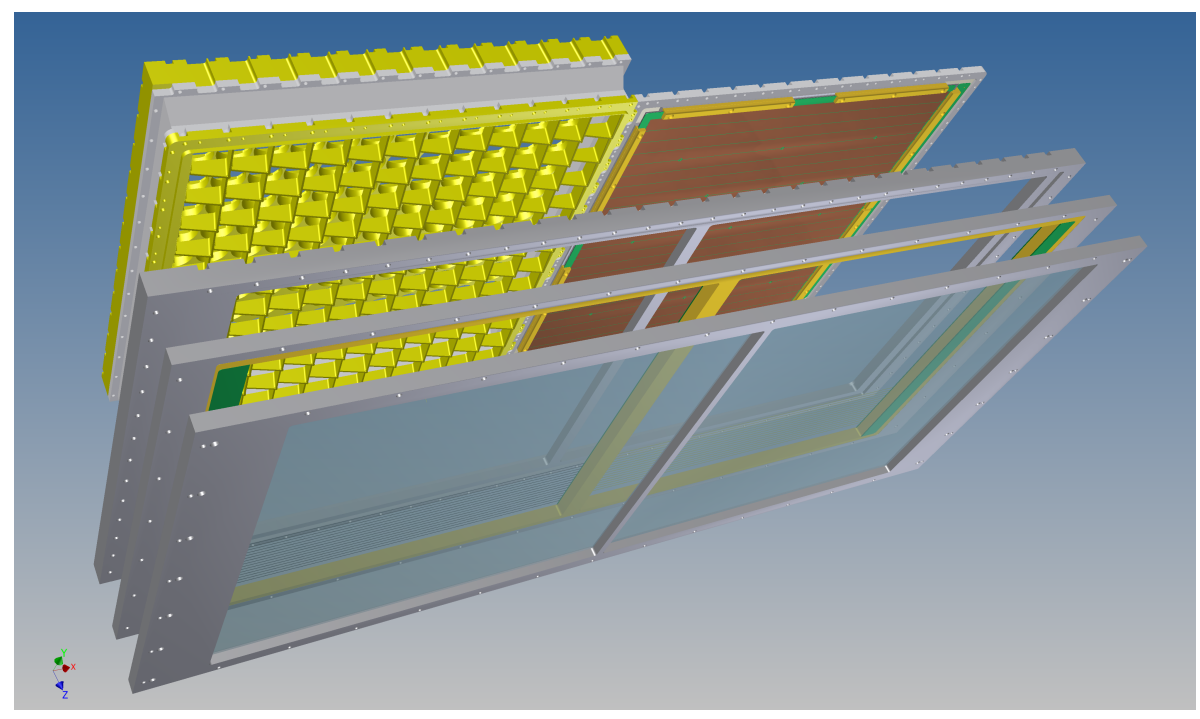

Figure 29. Exploded view of the foreseen structure of the upgraded COMPASS RICH-1 PDs.

\section{References}

[1] COMPASS collaboration, COMPASS: a proposal for a common muon and proton apparatus for structure and spectroscopy, CERN/SPSLC/96-14, SPSLC/P-297 (1996); COMPASS collaboration, COMPASS: Common Muon and Proton Apparatus for Structure and Spectroscopy, CERN/SPSLC/96-30, SPSLC/P-297-Add-1 (1996).

[2] COMPASS collaboration, COMPASS-II Proposal, CERN/SPSC/2010-014, SPSC-P-340 (2010); COMPASS collaboration, COMPASS-II Proposal: Questions \& Answers, CERN/SPSC/2010-022, SPSC-M-772 (2010).

[3] P. Abbon et al., The COMPASS experiment at CERN, Nucl. Instrum. Meth. A 577 (2007) 455.

[4] E. Albrecht et al., COMPASS RICH-1, Nucl. Instrum. Meth. A 502 (2003) 112.

[5] E. Albrecht et al., First performances of COMPASS RICH-1, Nucl. Instrum. Meth. A 518 (2004) 586.

[6] E. Albrecht et al., Status and characterisation of COMPASS RICH-1, Nucl. Instrum. Meth. A 553 (2005) 215.

[7] P. Abbon et al., The experience of building and operating COMPASS RICH-1, Nucl. Instrum. Meth. A 639 (2011) 15.

[8] E. Albrecht et al., The radiator gas and the gas system of COMPASS RICH-1, Nucl. Instrum. Meth. A 502 (2003) 266.

[9] S. Dalla Torre et al., A study of the RICH gas refractive index, Nucl. Instrum. Meth. A 639 (2011) 271.

[10] E. Albrecht et al., VUV absorbing vapours in n-perfluorocarbons, Nucl. Instrum. Meth. A 510 (2003) 262.

[11] E. Albrecht et al., The mirror system of COMPASS RICH-1, Nucl. Instrum. Meth. A 502 (2003) 236.

[12] S. Costa et al., CLAM, a continuous line alignment and monitoring method for RICH mirrors, Nucl. Instrum. Meth. A 553 (2005) 135.

[13] E. Albrecht et al., On-line mirror alignment monitoring method for COMPASS RICH-1, Nucl. Instrum. Meth. A 595 (2008) 194. 
[14] E. Albrecht et al., Mirror alignment control for COMPASS RICH-1 detector, Nucl. Instrum. Meth. A 639 (2011) 219.

[15] G. Baum et al., The COMPASS RICH project, Nucl. Instrum. Meth. A 433 (1999) 207.

[16] F. Piuz, Ring Imaging CHerenkov systems based on gaseous photo-detectors: trends and limits around particle accelerators, Nucl. Instrum. Meth. A 502 (2003) 76.

[17] A. Braem et al., Results from the ageing studies of large CsI photocathodes exposed to ionizing radiation in a gaseous RICH detector, Nucl. Instrum. Meth. A 553 (2005) 187.

[18] H. Hoedlmoser et al., Long term performance and ageing of CsI photocathodes for the ALICE/HMPID detector, Nucl. Instrum. Meth. A 574 (2007) 28.

[19] G. Baum et al., BORA: a front end board, with local intelligence, for the RICH detector of the Compass Collaboration, Nucl. Instrum. Meth. A 433 (1999) 426.

[20] G. Baum et al., The COMPASS RICH-1 read-out system, Nucl. Instrum. Meth. A 502 (2003) 246.

[21] P. Abbon et al., Fast readout of the COMPASS RICH CsI-MWPC photon chambers Nucl. Instrum. Meth. A 567 (2006) 104.

[22] M.J. French et al., Design and results from the APV25, a deep sub-micron CMOS front-end chip for the CMS tracker, Nucl. Instrum. Meth. A 466 (2001) 359.

[23] M. Alexeev et al., Long term experience with CsI photocathodes in gas photon detectors, 2014 JINST 9 P01006.

[24] P. Abbon et al., Particle identification with COMPASS RICH-1, Nucl. Instrum. Meth. A 631 (2011) 26.

[25] P. Abbon et al., Design and construction of the fast photon detection system for COMPASS RICH-1, Nucl. Instrum. Meth. A 616 (2010) 21.

[26] M. Alekseev et al., Studies for a fast RICH, Nucl. Instrum. Meth. A 553 (2005) 53.

[27] P. Abbon et al., Design and status of COMPASS FAST-RICH, Nucl. Instrum. Meth. A 567 (2007) 114.

[28] P. Abbon et al., Fast photon detection for COMPASS RICH-1, Nucl. Instrum. Meth. A 572 (2007) 419.

[29] P. Abbon et al., Fast photon detection for particle identification with COMPASS RICH-1, Nucl. Instrum. Meth. A 580 (2007) 906.

[30] P. Abbon et al., The fast photon detection system of COMPASS RICH-1, Nucl. Instrum. Meth. A 581 (2007) 419.

[31] P. Abbon et al., The characterisation of the multianode photomultiplier tubes for the RICH-1 upgrade project at COMPASS, Nucl. Instrum. Meth. A 595 (2008) 177.

[32] P. Abbon et al., Optical telescopes for COMPASS RICH-1 up-grade, Czech. J. Phys. Suppl. F 56 (2006) 315 .

[33] P. Abbon et al., Hartmann test of the COMPASS RICH-1 Optical telescopes, Czech. J. Phys. Suppl. F 56 (2006) 323.

[34] J. Hartmann, Bemerkungen über den Bau und die Justirung von Spektrographen, Z. Instrumentenkd. 20 (1900) 47.

[35] L.J. Golden, Dynamic Hartmann test, Appl. Opt. 14 (1975) 2391.

[36] P. Abbon et al., Read-out electronics for fast photon detection with COMPASS RICH-1, Nucl. Instrum. Meth. A 587 (2008) 371.

[37] P. Abbon et al., The fast readout system for the MAPMTs of COMPASS RICH-1, Nucl. Instrum. Meth. A 595 (2008) 204. 
[38] M.Chiosso et al., A full custom front-end ASIC Prototype "CMAD" for COMPASS-RICH-1 particle detector system, IEEE Nucl. Sci. Symp. Conf. Rec. 2008 (2008) 1495.

[39] F. Gonnella and M. Pegoraro, The MAD, a Full Custom ASIC for the CMS Barrel Muon Chambers Front End Electronics, CERN-LHCC-2001-034 (2001), pg. 204.

[40] H. Fischer et al., The COMPASS Data Acquisition System, IEEE Trans. Nucl. Sci. 49 (2002) 443; H. Fischer et al., Implementation of the dead-time free F1 TDC in the COMPASS detector readout, Nucl. Instrum. Meth. A 461 (2001) 507.

[41] P. Abbon et al., Pattern recognition and PID for COMPASS RICH-1, Nucl. Instrum. Meth. A 595 (2008) 233.

[42] T. Ypsilantis and J. Seguinot, Theory of ring imaging Cherenkov counters, Nucl. Instrum. Meth. A 343 (1994) 30.

[43] M. Alexeev et al., The quest for a third generation of gaseous photon detectors for Cherenkov imaging counters, Nucl. Instrum. Meth. A 610 (2009) 174.

[44] M. Alexeev et al., THGEM based photon detector for Cherenkov imaging applications, Nucl. Instrum. Meth. A 617 (2010) 396.

[45] M. Alexeev et al., Micropattern gaseous photon detectors for Cherenkov imaging counters, Nucl. Instrum. Meth. A 623 (2010) 129.

[46] M. Alexeev et al., Development of THGEM-based photon detectors for Cherenkov Imaging Counters, 2010 JINST 5 P03009.

[47] M. Alexeev et al., Progress towards a THGEM-based detector of single photons, Nucl. Instrum. Meth. A 639 (2011) 130.

[48] M. Alexeev et al., Detection of single photons with THickGEM-based counters, Nucl. Instrum. Meth. A 695 (2012) 159.

[49] F. Sauli, GEM: A new concept for electron amplification in gas detectors, Nucl. Instrum. Meth. A 386 (1997) 531.

[50] Y. Giomataris and G. Charpak, A hadron-blind detector, Nucl. Instrum. Meth. A 310 (1991) 589.

[51] W. Anderson et al., Design, construction, operation and performance of a Hadron Blind Detector for the PHENIX experiment, Nucl. Instrum. Meth. A 646 (2011) 35.

[52] P. Jeanneret, Time Projection Chambers and detection of neutrinos, Ph.D. Thesis, Neuchâtel University, Neuchâtel Switzerland (2001).

[53] L. Periale et al., Detection of the primary scintillation light from dense $\mathrm{Ar}, \mathrm{Kr}$ and Xe with novel photosensitive gaseous detectors, Nucl. Instrum. Meth. A 478 (2002) 377.

[54] P.S. Barbeau et al., Towards Coherent Neutrino Detection Using Low-Background Micropattern Gas Detectors, IEEE Trans. Nucl. Sci. 50 (2003) 1285.

[55] R. Chechik et al., Thick GEM-like hole multipliers: properties and possible applications, Nucl. Instrum. Meth. A 535 (2004) 303.

[56] Y. Giomataris et al., MICROMEGAS: a high-granularity position-sensitive gaseous detector for high particle-flux environments, Nucl. Instrum. Meth. A 376 (1996) 29.

[57] M. Alexeev et al., THGEM-based photon detectors for the upgrade of COMPASS RICH-1, Nucl. Instrum. Meth. A 732 (2013) 264. 